\title{
Gauge Invariance, Geometry and Arbitrage
}

\author{
Samuel E. Vázquez \\ Perimeter Institute for Theoretical Physics, \\ 31 Caroline St. N., Waterloo, ON, Canada N2L 2Y5 \\ Email: svazquez@perimeterinstitute.ca \\ Simone Farinelli, \\ UBS Group Risk Methodology \\ P.O. Box, Pelikanstrasse 6/8, CH-8098, Zurich \\ Email: simone.farinelli@ubs.com
}

August 21, 2009 


\begin{abstract}
In this work, we identify the most general measure of arbitrage for any market model governed by Itô processes. We show that our arbitrage measure is invariant under changes of numéraire and equivalent probability. Moreover, such measure has a geometrical interpretation as a gauge connection. The connection has zero curvature if and only if there is no arbitrage. We prove an extension of the Martingale pricing theorem in the case of arbitrage. In our case, the present value of any traded asset is given by the expectation of future cash-flows discounted by a line integral of the gauge connection. We develop simple strategies to measure arbitrage using both simulated and real market data. We find that, within our limited data sample, the market is efficient at time horizons of one day or longer. However, we provide strong evidence for non-zero arbitrage in high frequency intraday data. Such events seem to have a decay time of the order of one minute.
\end{abstract}




\section{Introduction}

The no-arbitrage principle is the cornerstone of modern financial mathematics. Put it simply, an arbitrage opportunity allows an agent to make a non-risky profit with zero or negative net investment (see [DeSc08]). Under the no-arbitrage assumption 1 one can assign in a complete market a unique price to the derivative of any traded assets using the replicating portfolio method (see [MuRu07] or [CvZa04]). The no-arbitrage principle can also be shown to be equivalent to a weaker form of economic equilibrium (cf. [CvZa04]) and can therefore be seen as a form of market efficiency (see [Fam98, [Ma03]). It is then not surprising that most financial and economic literature is based on the no-arbitrage assumption.

Nevertheless, the no-arbitrage principle represents a very strong assumption about market dynamics which must be tested empirically. Even when the market participants use noarbitrage models, the ultimate price of any security which is traded in a centralized market is set by supply/demand and the complex dynamics of the order book. That is, "the market" sets the prices. It then makes sense to ask: how efficient are these final prices? In order to answer this question one needs a measure of arbitrage.

There is a large body of empirical studies on financial arbitrage. Most of these studies focus on measuring the "excess return" of particular trading strategies (see e.g. [JeTi93, GaGoRo06]). Other studies try to find violations to general no-arbitrage relations between option prices (see e.g. AcTi99]). However, there does not seem to be a consensus on whether the reported market "anomalies" are due to arbitrage, or simply to random fluctuations (see [Ma03], Fam98]). Part of the problem is that there seems to be no general measure of arbitrage which can be applied to any traded asset. One of the main goals of this paper is to define such measure.

The second goal of this paper is to provide a geometrical interpretation of the arbitrage measure. In particular, it has been speculated long ago by Ilinski [I197], [1101] and Young [Y099] that arbitrage should be viewed as the "curvature" of a gauge connection, in analogy to some physical theories. The fact that gauge theories are the natural language to describe economics was first proposed by Malaney and Weinstein in the context of the economic index problem [Ma96], We06]. The need for such mathematical language can easily be seen from the fact that prices are only relational. More precisely, let $X(t)=\left(X_{1}(t), X_{2}(t), \ldots\right)^{\dagger}$ be the price vector of all goods in the economy at time $t$, in some common unit (say USD). Since the measuring units are arbitrary, fundamental economic laws must be invariant under the

\footnotetext{
${ }^{1}$ In continuous time the no-arbitrage condition (NA) has to be sharpened to the no-free-lunch-withvanishing-risk condition (NFLVR). Only under the (NFLVR) the fundamental theorem of asset pricing can be proved, see [DeSc08. Therefore, in this paper, when referring to (NA) in continuous time, (NFLVR) is meant.
} 
transformations

$$
X(t) \rightarrow \Lambda(t) X(t)
$$

where $\Lambda(t)>0$ is a positive stochastic factor 2 . In physics, a (local) transformation such as Eq. (11) is known as a gauge transformation. These represents a redundancy of our description of the economic system. The laws of the economy should be gauge invariant. The need for a gauge theoretical approach to economics was highlighted recently in [Sm09]. The role of gauge invariance in option pricing has been studied in [HoNe99a], HoNe99b], HoNe00] and [HoNeVe01]. For an unrelated use of differential geometric methods in (no-arbitrage) option pricing see [La08]. A recent proposal for a gauge connection in finance, and its relation to arbitrage, was presented in [Far08], Far09a and Far09b. In fact, we will show that the curvature of the gauge connection proposed in [Far08, Far09a and Far09b] is equivalent to our arbitrage measure.

In physics, curvature is a gauge invariant measure of the path dependency of some physical process. For example, readers familiar with electrodynamics might recall the vector potential $A_{\mu}$, where $\mu=0,1,2,3$ label the space-time directions. In differential geometry for theoretical physics, $A_{\mu}$ is known as a gauge connection. Now consider a charged particle which is traveling along some trajectory in space-time $x^{\mu}(s), s \in[0,1]$. The interaction of this particle with the gauge potential is proportional to the line integral, $\int_{\gamma} A:=\sum_{\mu} \int_{0}^{1} A_{\mu}(x(s)) \dot{x}^{\mu}(s) d s$. Now suppose that we make an infinitesimal change in the path of the particle $\delta x^{\mu}(s)$, keeping the boundary conditions fixed: $\delta x^{\mu}(0)=\delta x^{\nu}(1)=0$. The interaction changes by $\delta\left(\int_{\gamma} A\right)=\sum_{\mu, \nu} \int_{0}^{1} \delta x^{\mu}(s) \dot{x}^{\nu}(s) F_{\mu \nu}$, where $F_{\mu \nu}=\partial_{\mu} A_{\nu}-\partial_{\nu} A_{\mu}$ is known as the curvature of $A$. Therefore, we see that for zero curvature $F_{\mu \nu}=0$, the line integral $\int_{\gamma} A$ is independent of the path $\gamma$. Moreover, note that the curvature is invariant under a gauge transformation of the form $A_{\mu} \rightarrow A_{\mu}+\partial_{\mu} \Lambda$, where $\Lambda$ is any function of space-time.

We will find a very similar construction in the case of mathematical finance. In particular, we will show that the arbitrage curvature defined in this paper measures the path dependency of the present value of a self-financing portfolio of traded assets with fixed final payoff. The no-arbitrage principle is then equivalent to a zero-curvature condition. In analogy with the electromagnetic curvature $F$, we expect that any measure of arbitrage should be invariant under the gauge transformation in Eq. (11). Moreover, the fundamental theorem of asset pricing states that the no-arbitrage principle is equivalent to the existence of a probability measure with respect to which asset prices expressed in terms of a numéraire are Martingales [MuRu07]. Therefore, we expect that any measure of arbitrage should also be invariant under a change of probability. These are, in fact, two very important properties that will characterize our arbitrage measure.

\footnotetext{
${ }^{2}$ For example, $\Lambda(t)$ can be the EUR/USD exchange rate.
} 
This paper takes a "macroscopic" or phenomenological approach to arbitrage. More precisely, we will study arbitrage from the point of view of general stochastic models. We do not address the question of what is causing the arbitrage. Our main goal is to identify the gauge invariant financial observables that indicate an arbitrage opportunity. We believe that a proper understanding of such quantity, including its geometrical interpretation, is a first step towards a theory of non-equilibrium economics. Moreover, our methods can be applied to the construction of profitable trading strategies. Our main assumption is that the prices of all financial instruments can be described by Itô processes. Moreover, we ignore transaction costs. 3 .

The organization and main results of the paper are the following. In section 2 we present the class of models that we use in the rest of the paper. They are very general and include the case of stocks, bonds and commodities, and more complicated derivative products. We decompose the dynamics of these models in terms of their gauge transformation properties with respect to Eq. (1). We identify the gauge invariants and show that they represent an obstruction to the existence of a Martingale probability measure. We conclude section 2 with an example with three assets, and we derive a modified non-linear Black Scholes equation with arbitrage.

In section 3, we give a geometrical interpretation to the gauge invariant quantities defined in section 2. Our main goal is to identify the stochastic gauge invariants of section 2 with the curvature of a gauge connection. We begin with a review of the Malaney-Weinstein connection [Ma96], We06], which is done in the context of differentiable economic paths. In section 3.1, we generalize the Malaney-Weinstein construction to stochastic processes and prove an asset pricing theorem. The main result of this section is that the present value of any self-financing portfolio of traded assets is given by the conditional expectation of future cash-flows, discounted by a line integral of the Malaney-Weinstein gauge connection. We show how the value functions of different portfolios replicating the same contingent claim are related to the arbitrage curvature. Finally, we show that the gauge connection recently proposed in [Far08], Far09a] and [Far09b], is equivalent to the present construction. Readers interested in only the arbitrage measure and the detection techniques, can skip section 3. None of the results of section 4 require an understanding of the geometry of arbitrage. In section 4 we develop a simple algorithm to measure the arbitrage curvature using financial data. We explain the main sources of error in such measurement. The algorithm is applicable to any financial instrument. We provide examples with financial data of stock indexes and index futures. We find that, on long time scales, the market is very efficient. However, we

\footnotetext{
${ }^{3}$ Note that, as pointed out in [ShVi97], many possible arbitrage opportunities disappear once one takes into account market frictions such as transaction costs. Therefore, it is important to keep in mind that even when we measure a non-trivial curvature in the market, it does not mean that it can always be exploited in a practical trading strategy.
} 
provide strong evidence for non-zero curvature fluctuations at short time scales in the order of one minute. We conclude in section 5.

\section{Stochastic Models and Gauge Invariance}

Let $\mathcal{M}=\{0,1,2, \ldots, N-1\}$ be the set of all traded securities at any point in time in the market. We will use greek indices $\mu, \nu, \ldots$ to label members of the set $\mathcal{M}$. We denote the price of security $\mu \in \mathcal{M}$ by $X_{\mu}$. Our main assumption is that the dynamics of all such securities is described by Itô processes of the form [So06] and [Sh00]:

$$
d X_{\mu}:=X_{\mu}\left(\alpha_{\mu} d t+\sum_{a} \sigma_{\mu}^{a} d W_{a}\right), \quad \forall \mu \in \mathcal{M}
$$

where $W_{a}$ are standard Brownian motion such that $W_{a}(t)-W_{a}(0)$ are independently and normally distributed random variables with,

$$
\mathbb{E}\left[W_{a}(t)-W_{a}(0)\right]=0, \quad \operatorname{Cov}\left[W_{a}(t)-W_{a}(0), W_{b}(t)-W_{b}(0)\right]=t \delta_{a b} .
$$

We make no assumptions about the number of Brownian terms, and hence the completeness of the market. The set of Brownian motions $\left\{W_{a}\right\}$ represent all the randomness in the market. Therefore, they induce a natural filtration $\mathcal{F}=\left(\mathcal{F}_{t}\right)_{t \geq 0}$ for our probability space. The coefficients $\alpha_{\mu}$ and $\sigma_{\mu}^{a}$ can also be stochastic processes adapted 4 to the filtration $\mathcal{F}$. However, they are assumed to satisfy suitable conditions to ensure the existence of the price processes $X_{\mu}$ (see [LaLa06]). This class of models is very general and includes stocks, bonds, options, etc. Moreover, the case of fat tails in the distribution of returns is also included, since this is known to be generated by stochastic volatilities $\sigma_{\mu}^{a}$.

Looking back at Eq. (2), we can see that the tangent space $d X_{\mu}$ has a natural decomposition into the directions which contain all the randomness $\left(\sum_{a} \sigma_{\mu}^{a} d W_{a}\right)$ and those orthogonal to it. Therefore, we will make the following decomposition of the drift term in Eq. (2):

$$
\alpha_{\mu}=\alpha+\sum_{a} \beta^{a} \hat{\sigma}_{\mu}^{a}+\sum_{A \in \mathcal{N}} \alpha^{A} J_{\mu}^{A}
$$

\footnotetext{
${ }^{4}$ In simple terms, a process $p$ adapted to $\mathcal{F}$ means that it does not depend on future values of the Brownian motion. In other words, $p(t)$ can only depend on $\left\{W_{a}(s)\right\}$ up to time $s \leq t$.
} 
where $\mathcal{N}$ is the space spanned by basis vectors $J^{A}:=\left[J_{0}^{A}, \ldots, J_{N-1}^{A}\right]^{\dagger}$ such that

$$
\sum_{\mu} J_{\mu}^{A} J_{\mu}^{B}=\delta^{A B}, \quad \sum_{\mu} J_{\mu}^{A}=0, \quad \sum_{\mu} J_{\mu}^{A} \sigma_{\mu}^{a}=0, \quad \forall a
$$

and

$$
\alpha:=\frac{1}{N} \sum_{\mu} \alpha_{\mu}, \quad \alpha^{A}:=\sum_{\mu} J_{\mu}^{A} \alpha_{\mu} \quad \hat{\sigma}_{\mu}^{a}:=\sigma_{\mu}^{a}-\sigma^{a}, \quad \sigma^{a}:=\frac{1}{N} \sum_{\mu} \sigma_{\mu}^{a} .
$$

We will refer to $\mathcal{N}$ as the null space of the market. Note that this space is orthogonal to all the randomness in the tangent space $d X_{\mu}$. However, we need to remember that $\mathcal{N}$ might be trivial. The definition of $\beta^{a}$ in Eq. (4) is not unique if the vectors $\hat{\sigma}_{\mu}^{a}:=\left[\hat{\sigma}_{0}^{a}, \ldots, \hat{\sigma}_{N-1}^{a}\right]^{\dagger}$ are linearly dependent. This is the case of, for example, an incomplete market with more Brownian motions than traded securities. Moreover, $\alpha^{A}$ is unique up to rotations in the null space. As we will see, the quantities $\alpha^{A}$ are the unique gauge invariant measures of arbitrage. The two main goals of this paper are to give a geometric interpretation to the parameters $\alpha^{A}$, and to set up a procedure to measure them using financial data.

Since prices are relative and only reflect an exchange rate between two products, the units used to measure $X_{\mu}$ are arbitrary. Therefore, the dynamics of the market must be invariant under a change of measuring units. In mathematical finance, this is known as a change of numéraire [MuRu07], and it can be interpreted as a gauge transformation,

$$
X_{\mu}(t) \rightarrow \Lambda(t) X_{\mu}(t)
$$

where $\Lambda$ is a positive stochastic process which is adapted to the filtration $\mathcal{F}$. Another symmetry, which is special to the particular models of Eq. (2), is a transformation of the probability measure. This is not really a gauge symmetry, but corresponds rather to a change of variables of the form $W_{a}(t) \rightarrow W_{a}(t)+\int^{t} \delta \beta^{a}(s) d s$.

Our next task is to study the transformation properties of the different terms in Eqs. (2) and (4). The following result follows.

Proposition 2.1: Consider a change of numéraire of the form $X_{\mu} \rightarrow \Lambda X_{\mu}$, where $\Lambda$ is a positive stochastic process adapted to the filtration $\mathcal{F}$, and $d \Lambda:=\Lambda\left(\delta \alpha d t+\sum_{a} \delta \sigma^{a} d W_{a}\right)$. Then, the coefficients of the Itô processes, Eqs. (2) and (4), transform as

$$
\alpha \rightarrow \alpha+\delta \alpha+\sum_{a} \sigma^{a} \delta \sigma^{a}, \quad \beta^{a} \rightarrow \beta^{a}+\delta \sigma^{a}, \quad \sigma^{a} \rightarrow \sigma^{a}+\delta \sigma^{a}
$$

Finally, under a transformation of the probability measure given by the Radon-Nykodym derivative $d \mathbb{P} / d \mathbb{P}^{*}=\exp \left(-\int^{t} \sum_{a} \delta \beta^{a} d W_{a}-\frac{1}{2} \int^{t} \sum_{a}\left(\delta \beta^{a}(s)\right)^{2} d s\right)$, we have the mapping of 
standard Brownian motions

$$
W_{a}(t) \rightarrow W_{a}(t)+\int^{t} \delta \beta^{a}(s) d s
$$

and

$$
\alpha \rightarrow \alpha+\sum_{a} \sigma^{a} \delta \beta^{a}, \quad \beta^{a} \rightarrow \beta^{a}+\delta \beta^{a}
$$

In particular, it follows that $\hat{\sigma}_{\mu}^{a}, \alpha^{A}$ and $J_{\mu}^{A}$ are invariant under such transformations.

Proof: The result in Eq. (8) above follow from a simple application of Itô rule to the product $X_{\mu}^{\prime}:=\Lambda X_{\mu}$ :

$$
\begin{aligned}
d X_{\mu}^{\prime}= & d \Lambda X_{\mu}+\Lambda d X_{\mu}+d\left\langle\Lambda, X_{\mu}\right\rangle \\
= & X_{\mu}^{\prime}\left[\left(\alpha+\delta \alpha+\sum_{a} \sigma^{a} \delta \sigma^{a}+\sum_{a}\left(\beta^{a}+\delta \sigma^{a}\right) \hat{\sigma}_{\mu}^{a}+\sum_{A \in \mathcal{N}} \alpha^{A} J_{\mu}^{A}\right) d t\right. \\
& \left.+\sum_{a}\left(\hat{\sigma}_{\mu}^{a}+\sigma^{a}+\delta \sigma^{a}\right) d W_{a}\right],
\end{aligned}
$$

where $d\left\langle\Lambda, X_{\mu}\right\rangle=d t \Lambda X_{\mu} \sum_{a} \delta \sigma^{a} \sigma_{\mu}^{a}$ is the differential of the quadratic variation. The transformation in Eq. (10) follows from a simple differentiation of $W_{a}$ in Eq. (2):

$$
\begin{aligned}
d X_{\mu}= & X_{\mu}\left[\left(\alpha+\sum_{a} \sigma^{a} \delta \beta^{a}+\sum_{a}\left(\beta^{a}+\delta \beta^{a}\right) \hat{\sigma}_{\mu}^{a}+\sum_{A \in \mathcal{N}} \alpha^{A} J_{\mu}^{A}\right) d t\right. \\
& \left.+\sum_{a}\left(\hat{\sigma}_{\mu}^{a}+\sigma^{a}\right) d W_{a}^{*}\right]
\end{aligned}
$$

where we defined $W_{a}(t)=W_{a}^{*}(t)+\int^{t} \delta \beta^{a}(s) d s$. Note that both $\alpha^{A}$ and $J^{A}$ are unchanged by these gauge transformations. In particular, suppose that $\sum_{\mu} J_{\mu}^{A} \sigma_{\mu}^{a}=0$. Then, it follows from Eq. (5) that $\sum_{\mu} J_{\mu}^{A}\left(\sigma_{\mu}^{a}+\delta \sigma^{a}\right)=\sum_{\mu} J_{\mu}^{A} \sigma_{\mu}^{a}=0$.

So far we have taken the existence of the basis vectors $J^{A}$ for granted. A constructive procedure to find such basis, if non-trivial, is given by the following proposition.

Proposition 2.2: Let $\Omega$ be the symmetric and real $N \times N$ matrix with component $\Omega_{\mu \nu}=$ $\sum_{a} \sigma_{\mu}^{a} \sigma_{\nu}^{a}$, where $N=\operatorname{dim}(\mathcal{M})$. Moreover, define $U$ as the matrix of all ones, e.g. $U_{\mu \nu}=1$, $\forall \mu, \nu \in \mathcal{M}$. Then, the matrix $G$ defined by

$$
G=\Omega-\frac{1}{N}(U \Omega+\Omega U)+\frac{1}{N^{2}} \operatorname{Tr}(U \Omega) U
$$


is gauge invariant. Let $\mathcal{N}_{G}$ be the null space of matrix $G$ such that $\sum_{\mu} J_{\mu}=0$ for any nontrivial $J \in \mathcal{N}_{G}$. Then $\mathcal{N}_{G}=\mathcal{N}$. In particular, the space $\mathcal{N}_{G}$ is spanned by the orthonormal zero-modes of $G$ which are orthogonal to the vector $J=(1,1, \ldots, 1)^{\dagger}$.

Proof: First we need to prove that the space of vectors $J$ such that $J^{\dagger} \sigma^{a}=0, \forall a$, is in one-to-one correspondence with the zero modes of $\Omega$ : $\Omega J=0$. Obviously, if $J^{\dagger} \sigma^{a}=0$, it follows that $J$ is also a zero-mode of $\Omega$. To prove the converse, suppose that $\Omega J=0$, but $J^{\dagger} \sigma^{a}=\lambda^{a}$, where $\lambda^{a} \neq 0$ for at least one value of $a$. Then, $0=J^{\dagger} \Omega J=\sum_{a}\left(\lambda^{a}\right)^{2}$, which can only be true if $\lambda^{a}=0 \forall a$.

Now we turn our attention to the matrix $G$, defined in Eq. (13). Using the gauge transformation $\sigma_{\mu}^{a} \rightarrow \sigma_{\mu}^{a}+\delta \sigma^{a}$, we can see that $\Omega$ transforms as

$$
\Omega_{\mu \nu} \rightarrow \Omega_{\mu \nu}+\sum_{a} \delta \sigma^{a}\left(\sigma_{\mu}^{a}+\sigma_{\nu}^{a}\right)+\sum_{a}\left(\delta \sigma^{a}\right)^{2} .
$$

It is then straightforward to verify the gauge invariance of the matrix $G$. Next we recall that the space $\mathcal{N}$ is spanned by (non-trivial) orthonormal zero-modes of $\Omega$ such that they also satisfy $\sum_{\mu} J_{\mu}=0$. One can define a similar space $\mathcal{N}_{G}$ for $G$. It is easy to verify that any vector $J \in \mathcal{N}$ is also a vector in $\mathcal{N}_{G}$. On the other hand, for any vector $J^{\prime} \in \mathcal{N}_{G}$, it follows from Eq. (13) that $\Omega J^{\prime}=0$. Thus, we have proven that $\mathcal{N}=\mathcal{N}_{G}$.

It is easy to verify that $\sum_{\mu} G_{\mu \nu}=0$. Therefore, the vector $J=(1,1, \ldots, 1)^{\dagger}$ is a particular zero-mode of G. Now take any other zero-mode of $G$, call it $J^{\prime}$, which is orthogonal to $J$. It follows that, $0=J^{\dagger} J^{\prime}=\sum_{\mu} J_{\mu}^{\prime}$. Therefore, $J^{\prime} \in \mathcal{N}_{G}=\mathcal{N}$. This completes the proof.

So far we have talked about the full set of securities of the market. However, it is clear that the decomposition in Eq. (4) can be done for any subset of the market. That is, suppose we observe a subset of the prices $X_{i}, i \in \mathcal{S} \subset \mathcal{M}$. Moreover, suppose we find that within this subset one still can find some zero-modes $J^{A}$ obeying, $\sum_{i} J_{i}^{A} \sigma_{i}^{a}=0, \forall a$ and $\sum_{i} J_{i}^{A}=0$. One can then easily lift these vectors to the full set $\mathcal{M}$ by taking $J_{\mathcal{M}}^{A}=\left(J^{A}, \overrightarrow{0}\right)$. This represents a particular choice of basis in the null space $\mathcal{N}$. By observing a sub-sector of the market, we will only have access to some of the components of $\alpha^{A}$. For notational convenience, we will not distinguish between the full market and a subset of it in what follows.

Under the no-arbitrage assumption (see [MuRu07], [CvZa04]), it is always possible to find a common positive discount factor $\Lambda$ and an equivalent probability measure $\mathbb{P}^{*} \sim \mathbb{P}$ such that the discounted prices $\Lambda X_{\mu}$ are martingales: $\Lambda(t) X_{\mu}(t)=\mathbb{E}_{t}^{*}\left[\Lambda(T) X_{\mu}(T)\right]$, where $t \leq T$. This is known as the Martingale representation theorem (see [So06] and [Sh00]). In our language, this means that there is a gauge transformation mapping $X_{\mu}$ to $\mathbb{P}^{*}$-Martingales. In other words, if there is no arbitrage, price processes are gauge-equivalent to $\mathbb{P}^{*}$-Martingales for 
some probability measure $\mathbb{P}^{*}$. The result of the Martingale representation theorem can only be obtained if one is able to write $\int_{t}^{T} d\left(\Lambda X_{\mu}\right):=\int_{t}^{T} \gamma_{\mu}^{a} d W_{a}^{*}$ for some adapted process $\gamma_{\mu}^{a}$. The reason is that the stochastic integral $\int_{t}^{T} \gamma_{\mu}^{a} d W_{a}^{*}$ is a Martingale: $\mathbb{E}_{t}^{*}\left[\int_{t}^{T} \gamma_{\mu}^{a} d W_{a}^{*}\right]=0$. By proposition 2.2 there is neither a change of probability nor a choice of a positive discount factor for which the vector $\sum_{A \in \mathcal{N}} \alpha^{A} J^{A}$ is mapped to 0 (in contrast to $\alpha$ and all $\beta^{a}$ s which can indeed be made vanish). Therefore, it is easy to see that the term $\sum_{A \in \mathcal{N}} \alpha^{A} J^{A}$ parametrizes the obstruction to the existence of a Martingale probability measure for any discounted price process $\Lambda X_{\mu}$.

As $\alpha^{A}$ S are gauge invariant quantities, one expects that they should be observables. In the next section we will relate this quantity to a gauge connection and its curvature. In section 4 we will show that such quantity can indeed be observed, and we explain simple strategies to measure it. Before concluding this section, it is instructive to a study particular example with three assets.

\subsection{An Example}

Consider the case of three assets $X_{\mu}, \mu=0,1,2$, where $X_{0}$ is a savings account and $X_{1}, X_{2}$ are some other risky assets. All prices are measured in the same common units. We will assume only one Brownian motion. Therefore, the dynamics of the prices is described by

$$
\begin{aligned}
& d X_{0}=r X_{0} d t \\
& d X_{i}=X_{i}\left[\alpha_{i} d t+\sigma_{i} d W\right], \quad i=1,2 .
\end{aligned}
$$

For later convenience, we assume that the interest rate $r$ is deterministic. In order to do the decomposition in Eq. (4) we need to find a basis for the null space $\mathcal{N}$. In this case, since there is only one Brownian motion and two risky assets, there will be only one null direction. To calculate it, we start by identifying the $\Omega$ matrix:

$$
\Omega=\left(\begin{array}{ccc}
0 & 0 & 0 \\
0 & \sigma_{1}^{2} & \sigma_{1} \sigma_{2} \\
0 & \sigma_{1} \sigma_{2} & \sigma_{2}^{2}
\end{array}\right)
$$

One can now construct the $G$ matrix using Eq. (13). The explicit form of $G$ is not very illuminating. The unormalized eigenvectors of $G$ are found to be

$$
V_{1}=\left(\begin{array}{c}
2-\frac{3 \sigma_{1}}{\sigma_{1}+\sigma_{2}} \\
0 \\
0
\end{array}\right), \quad V_{2}=\left(\begin{array}{c}
-1+\frac{3 \sigma_{1}}{\sigma_{1}+\sigma_{2}} \\
1 \\
0
\end{array}\right), \quad V_{3}=\left(\begin{array}{c}
\frac{\sigma_{1}+\sigma_{2}}{\sigma_{1}-2 \sigma_{2}} \\
\frac{\sigma_{2}-2 \sigma_{1}}{\sigma_{1}-2 \sigma_{2}} \\
1
\end{array}\right)
$$


where

$$
G V_{1}=0, \quad G V_{2}=0, \quad G V_{3}=\frac{2}{3}\left(\sigma_{1}^{2}+\sigma_{2}^{2}-\sigma_{1} \sigma_{2}\right) V_{3} .
$$

In order to find a basis for the null space $\mathcal{N}$ defined in Eq. (5), we need to project $V_{1}$ or $V_{2}$ into the space orthogonal to the vector $J^{0}=(1,1, \ldots 1)^{\dagger}$. To do this, we define the projection matrix

$$
P_{U}:=\frac{1}{3} U, \quad P_{U}^{2}=P_{U},
$$

where $U$ is the $3 \times 3$ all-ones matrix. Note that $1-P_{U}$ projects into the space orthogonal to $J^{0}$. Our choice for the normalized null vector is then

$$
J=\frac{\left(\mathbf{1}-P_{U}\right) V_{1}}{\sqrt{\left[\left(\mathbf{1}-P_{U}\right) V_{1}\right]^{\dagger}\left(\mathbf{1}-P_{U}\right) V_{1}}}=\frac{1}{\sqrt{2} \sqrt{\sigma_{1}^{2}+\sigma_{2}^{2}-\sigma_{1} \sigma_{2}}}\left(\begin{array}{c}
\sigma_{1}-\sigma_{2} \\
\sigma_{2} \\
-\sigma_{1}
\end{array}\right) .
$$

It is easy to verify that $J$ obeys the properties given in Eq. (5).

We can now go back to the decomposition given in Eq. (4). Using Eqs. (15), we find

$$
\alpha=r-\beta \hat{\sigma}_{0}-\tilde{\alpha} J_{0}, \quad \sigma_{0}=0,
$$

where $\hat{\sigma}_{\mu}=\sigma_{\mu}-\frac{1}{3} \sum_{\nu=0}^{2} \sigma_{\nu}$, and $\tilde{\alpha}$ is the arbitrage vector $\alpha^{A}$, which in this case has only one component $\alpha^{1}:=\tilde{\alpha}$. Therefore, inserting Eqs. (21) into Eqs. (15), we can write the evolution equations as

$$
\begin{aligned}
& d X_{0}=r X_{0} d t \\
& d X_{1}=X_{1}\left[\left(r+\beta \sigma_{1}+\tilde{\alpha} \frac{2 \sigma_{2}-\sigma_{1}}{\sqrt{2} \sqrt{\sigma_{1}^{2}+\sigma_{2}^{2}-\sigma_{1} \sigma_{2}}}\right) d t+\sigma_{1} d W\right], \\
& d X_{2}=X_{2}\left[\left(r+\beta \sigma_{2}+\tilde{\alpha} \frac{\sigma_{2}-2 \sigma_{1}}{\sqrt{2} \sqrt{\sigma_{1}^{2}+\sigma_{2}^{2}-\sigma_{1} \sigma_{2}}}\right) d t+\sigma_{2} d W\right] .
\end{aligned}
$$

For $\tilde{\alpha}=0$, Eqs. (221) - (24) reduce to the familiar no-arbitrage Black-Scholes dynamics. As usual, $\beta$ is interpreted as the market price of risk. Note that in this example, both risky assets are exposed to the same market risk factor $W$. The volatility $\sigma_{i}$ measures the coupling to such risk. Under the no-arbitrage assumption, both assets should give the same expected return per unit of risk. This is $\beta$. However, we see that if $\tilde{\alpha} \neq 0, X_{1}$ and $X_{2}$ have different expected returns, even when they are exposed to the same risk. This discloses an arbitrage opportunity.

There is a very interesting consequence of Eqs. (22) - (24) when $X_{2}$ is any function of $X_{1}$, e.g. an option. For simplicity, consider the case where the only time dependence in $\tilde{\alpha}$ is 
of the form $\tilde{\alpha}=\tilde{\alpha}\left(t, X_{1}\right)$, where $\tilde{\alpha}\left(t, X_{1}\right)$ is a differentiable function of $t$ and $X_{1}$. Moreover, the interest rate $r$ is assumed to be deterministic. In this case one can derive a non-linear version of the Black Scholes equation with arbitrage. For ease of notation let $X_{1}:=X$. Under our assumptions we will have that $X_{2}=V(t, X)$. Then, using Itô rule we find

$$
\begin{aligned}
d V & =\partial_{t} V d t+\partial_{X} V d X+\frac{1}{2} \partial_{X}^{2} V d\langle X\rangle \\
& =V\left(\alpha_{2} d t+\sigma_{2} d W\right)
\end{aligned}
$$

where we identify

$$
\alpha_{2}=\frac{\partial_{t} V}{V}+\alpha_{1} X \frac{\partial_{X} V}{V}+\frac{1}{2} \sigma_{1}^{2} X^{2} \frac{\partial_{X}^{2} V}{V}, \quad \sigma_{2}=\sigma_{1} X \frac{\partial_{X} V}{V}
$$

Comparing Eq. (26) with Eq. (24) we find

$$
\alpha_{2}=r+\beta \sigma_{2}+\tilde{\alpha} \frac{\sigma_{2}-2 \sigma_{1}}{\sqrt{2} \sqrt{\sigma_{1}^{2}+\sigma_{2}^{2}-\sigma_{1} \sigma_{2}}}=\frac{\partial_{t} V}{V}+\alpha_{1} X \frac{\partial_{X} V}{V}+\frac{1}{2} \sigma_{1}^{2} X^{2} \frac{\partial_{X}^{2} V}{V}
$$

where from Eq. (23)

$$
\alpha_{1}=r+\beta \sigma_{1}+\tilde{\alpha} \frac{2 \sigma_{2}-\sigma_{1}}{\sqrt{2} \sqrt{\sigma_{1}^{2}+\sigma_{2}^{2}-\sigma_{1} \sigma_{2}}} .
$$

Therefore, after some algebra Eq. (27) becomes a modified non-linear Black Scholes partial differential equation:

$$
\partial_{t} V+r X \partial_{X} V+\frac{1}{2} \sigma_{1}^{2} X^{2} \partial_{X}^{2} V+\left(\sqrt{2} \tilde{\alpha}\left[1+\frac{X \partial_{X} V}{V}\left(\frac{X \partial_{X} V}{V}-1\right)\right]^{1 / 2}-r\right) V=0
$$

Note that for $\tilde{\alpha}=0$ this reduces to the familiar Black Scholes equation. The non-linear Black Scholes equation is a special case of the more general pricing theorem presented in section 3 .

It is important to remember that the arbitrage parameter, $\tilde{\alpha}$ in Eq. (29), can in general depend on time and the stock price. Therefore, in principle almost any deformation of the option price is possible. It follows that the Eq. (29) can be solved only if the arbitrage dynamics is known. For example, consider the case where we set

$$
\tilde{\alpha}:=\frac{1}{2^{3 / 2}}\left(\tilde{\sigma}_{1}^{2}-\sigma_{1}^{2}\right) \frac{X^{2} \partial_{X}^{2} V}{V\left[1+\frac{X \partial_{X} V}{V}\left(\frac{X \partial_{X} V}{V}-1\right)\right]^{1 / 2}}
$$

for some constant $\tilde{\sigma}_{1}^{2}$. Then, the option price obeys the usual Black-Scholes equation but 
with the "wrong" volatility:

$$
\partial_{t} V+r\left(X \partial_{X} V-V\right)+\frac{1}{2} \tilde{\sigma}_{1}^{2} X^{2} \partial_{X}^{2} V=0
$$

This is a simple example of the well-known volatility arbitrage.

\section{The Gauge Connection}

The application of differential geometric ideas in economics can be traced to the work of Malaney and Weinstein ([Ma96], We06]). It was found that the solution to the apparent discrepancy among different economic growth indices could be solved by the appropriate choice of a covariant derivative. Such derivative has the property that a self-financing basket of goods is seen as "constant". More technically, a self-financing basket is interpreted as being "parallel transported" along a one dimensional curve in the a base manifold spanned by prices and portfolio nominals. Then, there is a natural geometric index to measure the growth of such basket, which was shown to be identical to the so-called Divisa Index. It is very illuminating to review this construction to gain intuition about the relation between arbitrage and curvature. In what follows, all quantities are assumed to be deterministic and differentiable. We will return to the stochastic case in the next subsection.

A covariant derivative induces a connection one-form in the base space, and in [Ma96], [We06] this connection is given by

$$
A=\frac{\sum_{\mu} \phi_{\mu} d X_{\mu}}{\sum_{\nu} \phi_{\nu} X_{\nu}}
$$

where $\phi_{\mu}$ are the portofolio nominals, $V=\sum_{\mu} \phi_{\mu} X_{\mu}$, and the base space is parametrized by the coordinates $\left(t, \phi_{\mu}, X_{\mu}\right)$. Note that under a change of numéraire $X_{\mu} \rightarrow \Lambda(X) X_{\mu}$, the connection transforms as

$$
A \rightarrow A+d \Lambda
$$

This is the analog of the transformation rule of the vector potential in electrodynamics.

A self-financing portfolio can be seen as being parallel transported with the connection $A$ as

$$
\nabla_{\dot{\gamma}} V=\left.(d-A) V\right|_{\dot{\gamma}}=0
$$

where $\nabla_{\dot{\gamma}}$ is the covariant derivative along the trajectory $\gamma$. The solution to this equation is simply,

$$
\frac{V(T)}{V(t)}=e^{\int_{\gamma} A}:=D_{\gamma},
$$


where $\gamma$ is a particular self-financing trajectory $(s, \phi(s), X(s)), s \in[t, T]$, and $D_{\gamma}$ is known as the Divisa Index.

The dependence of $D_{\gamma}$ on the choice of curve $\gamma$ is parametrized by the curvature of the gauge connection, which is given by

$$
R=d A=\frac{1}{\left(\sum_{\mu} \phi_{\mu} X_{\mu}\right)^{2}} \sum_{\nu, \sigma}\left(\phi_{\nu} X_{\nu} d \phi_{\sigma} \wedge d X_{\sigma}-\phi_{\sigma} X_{\nu} d \phi_{\nu} \wedge d X_{\sigma}\right)
$$

Note that the curvature is invariant under a gauge transformation, as $d(A+d \Lambda)=d A$. In the approximation where economic agents are price takers, the price trajectory $X(t)$ is given exogenously, and we are only allowed to make changes in the portfolio nominals $\phi$. In other words, we can write $d X_{\mu}=\dot{X}_{\mu} d t$ in Eq. (36). One can then restrict the curvature to the submanifold corresponding to the $\left(t, \phi_{\mu}\right)$ coordinates. The induced curvature in this submanifold is given by

$$
R=\frac{1}{\left(\sum_{\mu} \phi_{\mu} X_{\mu}\right)^{2}} \sum_{\nu, \sigma} \phi_{\sigma} X_{\nu} X_{\sigma}\left(\frac{\dot{X}_{\nu}}{X_{\nu}}-\frac{\dot{X}_{\sigma}}{X_{\sigma}}\right) d \phi_{\nu} \wedge d t:=\sum_{\mu} R_{\mu, t} d \phi_{\mu} \wedge d t
$$

In this case, the path dependency of the Divisa Index, Eq. (35), can be written as,

$$
\delta_{\gamma} \log D_{\gamma}=\sum_{\mu} \int_{t}^{T} d s R_{\mu, t}(s) \delta \phi_{\mu}(s)
$$

where $\delta_{\gamma}$ represents a variation to the trajectory of the portfolio nominals. Therefore, we see that Eq. (35) is independent on the path $\gamma$ only if the price trajectories obey the zerocurvature condition $R_{\mu, t}=0 \Longrightarrow \dot{X}_{\mu}(t)=\alpha(t) X_{\mu}(t), \forall \mu$. The zero-curvature condition implies that the prices of all securities evolve by the same common inflation factor.

The relation between curvature and arbitrage goes as follow. Suppose that the prices obeyed the zero-curvature condition given above. It follows that, for any self-financing portfolio, we have

$$
V(T)=V(t) e^{\int_{\gamma} A}=V(t) e^{\int_{t}^{T} \alpha(s) d s},
$$

for $T \geq t$. In particular, if $V(t)=0$ it follows that $V(T)=0$. Therefore, it is not possible to make wealth without a positive initial investment. On the other hand, suppose that the curvature is not zero. Consider two portfolio trajectories $\gamma_{1}$ and $\gamma_{2}$ such that, say $D_{\gamma_{1}}>D_{\gamma_{2}}$ at some time $T \geq t$, for the same initial wealth $V_{\gamma_{1}}(t)=V_{\gamma_{2}}(t)>0$. Now construct the difference portfolio with nominals $\phi:=\phi_{1}-\phi_{2}$ and wealth function

$$
V=V_{\gamma_{1}}-V_{\gamma_{2}}
$$


Then, at time $T \geq t$ we have

$$
V(T)=\left(D_{\gamma_{1}}-D_{\gamma_{2}}\right) V_{\gamma_{1}}(t)>0
$$

while $V(t)=0$. In other words, we have made wealth out of nothing. In the next section we show how this construction carries over to the stochastic case.

\subsection{The Stochastic Gauge Connection}

In the previous section we illustrated the relation between curvature, path dependency and arbitrage, using the Malaney-Weinstein connection. However, this construction only works for differentiable economic trajectories in the base space $(\phi, X)$. Nevertheless, we have found a direct analog of the Malaney-Weinstein connection for Itô processes, which we summarize in the following theorem. In order to avoid technical complications, we restrict our attention to an economy on a finite interval of time $t \in[0, T]$.

Theorem 3.1: Consider any self-financing portfolio $V=\sum_{\mu} \phi_{\mu} X_{\mu}$, so that $d V=\sum_{\mu} \phi_{\mu} d X_{\mu}$. Then, there exist a (non-unique) equivalent probability measure $\mathbb{P}^{*} \sim \mathbb{P}$ under which the price processes obey

$$
d X_{\mu}=X_{\mu}\left[\left(\alpha^{*}+\sum_{A} \alpha^{A} J_{\mu}^{A}\right) d t+\sum_{a} \sigma_{\mu}^{a} d W_{a}^{*}\right] .
$$

Moreover, the present value of $V(t)$ given some final payoff $V(T), T \geq t$, is given by

$$
V_{\gamma}(t)=\mathbb{E}_{t}^{*}\left[V(T) e^{-\int_{\gamma} \Gamma}\right]
$$

where $\gamma$ is some self-financing trajectory, and $\Gamma$ is given by the expectation of the MalaneyWeinstein connection,

$$
\Gamma=\mathbb{E}_{t}^{*}\left[\frac{\sum_{\mu} \phi_{\mu} d X_{\mu}}{\sum_{\nu} \phi_{\nu} X_{\nu}}\right]=\frac{\sum_{\mu, A \in \mathcal{N}} \alpha^{A} J_{\mu}^{A} \phi_{\mu} X_{\mu}}{\sum_{\nu} \phi_{\nu} X_{\nu}} d t+\alpha^{*} d t
$$

Finally, the path dependency of the present value of the portfolio, with fixed final payoff, is parametrized by

$$
\delta V_{\gamma}(t)=-\sum_{\mu} \int_{t}^{T} d s \mathbb{E}_{t}^{*}\left[V(T) e^{-\int_{\gamma} \Gamma} \delta \phi_{\mu}(s) R_{\mu, t}(s)\right],
$$

where $R_{\mu, t}$ are the components of the curvature two-form defined in the reduced base space 
$(t, \phi)$,

$$
R=d \Gamma=\frac{1}{\left(\sum_{\mu} \phi_{\mu} X_{\mu}\right)^{2}} \sum_{\nu, \sigma, A \in \mathcal{N}} \alpha^{A} X_{\nu} X_{\sigma} \phi_{\sigma}\left(J_{\nu}^{A}-J_{\sigma}^{A}\right) d \phi_{\nu} \wedge d t:=\sum_{\mu} R_{\mu, t} d \phi_{\mu} \wedge d t
$$

Proof: We start by writing the portfolio return as

$$
d V=\sum_{\mu} \phi_{\mu} d X_{\mu}:=V\left(a d t+\sum_{a} b^{a} d W_{a}\right)
$$

where

$$
a=\frac{\sum_{\mu} \alpha_{\mu} \phi_{\mu} X_{\mu}}{\sum_{\nu} \phi_{\nu} X_{\nu}}, \quad b^{a}=\frac{\sum_{\mu} \sigma_{\mu}^{a} \phi_{\mu} X_{\mu}}{\sum_{\nu} \phi_{\nu} X_{\nu}}
$$

Now consider the combination $V^{\prime}:=\Lambda V$, where we take (c.f. Eq. (21) )

$$
d \Lambda=\Lambda\left[\left(-a+\sum_{a} b^{a} \beta^{a}\right) d t-\sum_{a} \beta^{a} d W_{a}\right]
$$

A simple application of Itô rule gives

$$
d V^{\prime}=V^{\prime} \sum_{a}\left(b^{a}-\beta^{a}\right) d W_{a} .
$$

It is well known that any stochastic integral of the form $\int_{0}^{t} \gamma d W_{a}$ is a Martingale [So06] and [Sh00. Therefore, we have

$$
V(t)=\mathbb{E}_{t}\left[V(T) e^{\int_{t}^{T} d \log \Lambda}\right]
$$

A further application of Itô rule gives

$$
d \log \Lambda=-\Gamma-\frac{1}{2} \sum_{a}\left(\beta^{a}\right)^{2} d t-\sum_{a} \beta^{a} d W_{a}
$$

where $\Gamma$ is defined in Eq. (44), with $\alpha^{*}:=\alpha-\sum_{a} \beta^{a} \sigma^{a}$.

Now consider making a change of probability measure such that $W_{a}:=W_{a}^{*}-\int^{t} \beta^{a}(s) d s$. It is easy to see that, under $\mathbb{P}^{*}$, the price processes will obey Eq. (42) of the theorem. Moreover, the Radon-Nykodým derivative is given by

$$
\frac{d \mathbb{P}}{d \mathbb{P}^{*}}=\exp \left[-\frac{1}{2} \sum_{a} \int_{t}^{T}\left(\beta^{a}(s)\right)^{2} d s+\sum_{a} \int_{t}^{T} \beta^{a} d W_{a}^{*}\right]
$$


Therefore, using Eqs. (52) and (53) in (51) we get

$$
\begin{aligned}
V(t) & =\mathbb{E}_{t}\left[V(T) e^{-\int_{\gamma} \Gamma-\frac{1}{2} \sum_{a} \int_{t}^{T}\left(\beta^{a}\right)^{2} d t-\sum_{a} \int_{t}^{T} \beta^{a} d W_{a}}\right] \\
& =\mathbb{E}_{t}^{*}\left[V(T) \frac{d \mathbb{P}}{d \mathbb{P}^{*}} e^{-\int_{\gamma} \Gamma+\frac{1}{2} \sum_{a} \int_{t}^{T}\left(\beta^{a}\right)^{2} d t-\sum_{a} \int_{t}^{T} \beta^{a} d W_{a}^{*}}\right] \\
& =\mathbb{E}_{t}^{*}\left[V(T) e^{-\int_{\gamma} \Gamma}\right]
\end{aligned}
$$

In order to prove that $\Gamma$ can be written as an expectation of the Malaney-Weinstein connection, we recall that

$$
\begin{aligned}
\Gamma & =\sum_{\mu} \frac{\phi_{\mu}(t) X_{\mu}(t)}{\sum_{\nu} \phi_{\nu}(t) X_{\nu}(t)}\left(\alpha^{*}+\sum_{A \in \mathcal{N}} \alpha^{A} J_{\mu}^{A}\right) d t \\
& =\lim _{\delta t \rightarrow 0} \sum_{\mu} \frac{\phi_{\mu}(t)}{\sum_{\nu} \phi_{\nu}(t) X_{\nu}(t)}\left(\frac{\mathbb{E}_{t}^{*}\left[X_{\mu}(t+\delta t)\right]-X_{\mu}(t)}{\delta t}\right) d t \\
& =\lim _{\delta t \rightarrow 0} \mathbb{E}_{t}^{*}\left[\sum_{\mu} \frac{\phi_{\mu}(t)}{\sum_{\nu} \phi_{\nu}(t) X_{\nu}(t)}\left(\frac{X_{\mu}(t+\delta t)-X_{\mu}(t)}{\delta t}\right) d t\right] \\
& =\mathbb{E}_{t}^{*}\left[\frac{\sum_{\mu} \phi_{\mu} d X_{\mu}}{\sum_{\nu} \phi_{\nu} X_{\nu}}\right] .
\end{aligned}
$$

The last result of the theorem, Eq. (45), follows simply by making a small change in the portfolio nominals, and keeping the boundary conditions on $V$ fixed.

Note that the curvature of $\Gamma$ is zero if and only if $\alpha^{A}=0$, which is equivalent to the no-arbitrage condition. Moreover, the probability measure $\mathbb{P}^{*}$ might not be unique, as the choice of $\beta^{a}$ in general is not. This also implies that $\alpha^{*}$ is not unique in general.

A special case of a self-financing portfolio is a portfolio containing just one base asset.

Corollary 3.2: For all assets in the market model $\mu \in \mathcal{M}$

$$
X_{\mu}(t)=\mathbb{E}_{t}^{*}\left[X_{\mu}(T) e^{-\int_{t}^{T}\left(\alpha^{*}+\sum_{A} \alpha^{A} J_{\mu}^{A}\right) d t^{\prime}}\right]
$$

In particular, under the no-arbitrage assumption $\alpha^{A}=0$, we recover the classic Martingale pricing theorem:

$$
X_{\mu}(t)=\mathbb{E}_{t}^{*}\left[X_{\mu}(T) e^{-\int_{t}^{T} \alpha^{*} d t^{\prime}}\right]
$$

In section 2.1 we derived a modified Black-Scholes equation for the case of three assets. Now we can use the result of Corollary 3.2 to prove a generalization of such equation. 
Consider the following vector of assets

$$
X=\left[X_{0}, X_{1}, \ldots, X_{n}, \Phi_{1}(\vec{X}, t), \ldots, \Phi_{m}(\vec{X}, t)\right]^{\dagger}
$$

We will label the components of this vector by $X_{\mu}, \mu=0,1, \ldots, n+m$. Moreover, we assume that $\Phi_{i}$ are smooth functions of the vector of underlying prices, $\vec{X}:=\left[X_{1}, \ldots, X_{n}\right]^{\dagger}$, and $d X_{0}=X_{0} r d t$ describes a savings account with deterministic interest rate $r$. The functions $\Phi_{i}(\vec{X}, t)$ describe a set of European-style contingent claims with final payoff $\Phi_{i}(\vec{X}(T), T)=$ $f_{i}(\vec{X}(T))$, for some fixed $T \geq t$. Finally, we need to assume that $\alpha^{A}$ are either deterministic or some function of the underlying prices $\vec{X}$. These assumptions ensure that the expectation

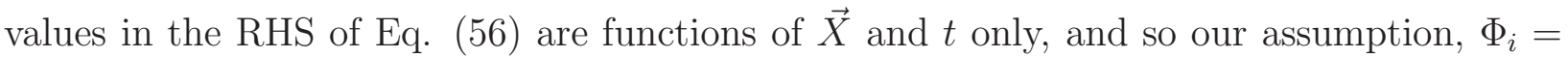
$\Phi_{i}(\vec{X}, t)$, is self-consistent. Under these assumptions we can prove the following corollary.

Corollary 3.3 (Modified Black-Scholes Equation): Under the assumptions given above, Corollary 3.2 implies that the European-style contingent claims $\Phi_{i}, i=1, \ldots, m$, obey the non-linear Black-Scholes equations

$\partial_{t} \Phi_{i}+\sum_{j=1}^{n}\left(\alpha^{*}+\sum_{A} \alpha^{A} J_{j}^{A}\right) X_{j} \partial_{j} \Phi_{i}-\left(\alpha^{*}+\sum_{A} \alpha^{A} J_{n+i}^{A}\right) \Phi_{i}+\frac{1}{2} \sum_{j, k=1}^{n} \Omega_{j k} X_{j} X_{k} \partial_{j} \partial_{k} \Phi_{i}=0$

with terminal conditions $\Phi_{i}(\vec{X}, T)=f_{i}(\vec{X})$. Moreover, $J^{A}=J^{A}(\vec{X}, t)$ are a basis for the null space $\mathcal{N}$ of the $(n+m+1) \times(n+m+1)$ matrix $\Omega$ with components $\Omega_{\mu \nu}=\sum_{a} \sigma_{\mu}^{a} \sigma_{\nu}^{a}$, where $\sigma_{i}^{a}, i=1, \ldots, n$ are the volatilities of the underlying securities, and we define $\sigma_{0}^{a}:=0$,

$$
\sigma_{n+i}^{a}:=\sum_{j=1}^{n} \sigma_{j}^{a} X_{j} \partial_{j} \log \Phi_{i}(\vec{X}, t), \quad i=1, \ldots, m
$$

and

$$
\alpha^{*}=r-\sum_{A} \alpha^{A} J_{0}^{A}
$$

Proof: The Eq. (59) of the corollary is a simple application of the Feynman-Kac theorem to Eqs. (56) (see [Sh00])). In order to calculate all components of the matrix $\Omega$, we remind the reader that the underlying prices $\vec{X}$ obey

$$
d X_{i}=X_{i}\left[\left(\alpha^{*}+\sum_{A} \alpha^{A} J_{i}^{A}\right) d t+\sum_{a} \sigma_{i}^{a} d W_{a}^{*}\right], \quad i=1, \ldots, n
$$


This implies that the stochastic part of $d \Phi_{i}$ is given by

$$
d \Phi_{i}(\vec{X}, t)=\Phi_{i}(\vec{X}, t) \sum_{j=1}^{n} X_{j} \partial_{j} \log \Phi_{i}(\vec{X}, t) \sigma_{j}^{a} d W_{a}^{*}+\ldots
$$

Therefore, the volatilities for the $X_{n+i}=\Phi_{i}$ securities are

$$
\sigma_{n+i}^{a}=\sum_{j=1}^{n} \sigma_{j}^{a} X_{j} \partial_{j} \log \Phi_{i}(\vec{X}, t)
$$

Moreover, since $X_{0}$ is a deterministic process, it follows that $\sigma_{0}^{a}=0$. In order to prove Eq. (61) of the corollary, we recall that the savings account obey $d X_{0}=r X_{0} d t$. This implies that $r=\alpha^{*}+\sum_{A} \alpha^{A} J_{0}^{A}$. This completes the proof.

The example of section 2.1 is a special case of the corollary 3.3 , with $n=m=1$. In this case there is only one null direction. We will use the notation $X_{1}:=X, \Phi_{1}:=V, \sigma_{1}^{1}:=\sigma_{1}$, and $\alpha^{1}:=\tilde{\alpha}$ in what follows. A choice for the basis of the null space was given in Eq. (20), which we repeat here for the convenience of the reader:

$$
J=\frac{1}{\sqrt{2} \sqrt{1+X \partial X \log V(X \partial X \log V-1)}}\left(\begin{array}{c}
1-X \partial X \log V \\
X \partial X \log V \\
-1
\end{array}\right)
$$

It follows that Eq. (159) becomes

$$
\begin{aligned}
0= & \partial_{t} V+\left(\alpha^{*}+\tilde{\alpha} J_{1}\right) X \partial_{X} V-\left(\alpha^{*}+\tilde{\alpha} J_{2}\right) V+\frac{1}{2} \sigma_{1}^{2} X^{2} \partial_{X}^{2} V \\
= & \partial_{t} V+\left(r+\tilde{\alpha}\left(J_{1}-J_{0}\right)\right) X \partial_{X} V-\left(r+\tilde{\alpha}\left(J_{2}-J_{0}\right)\right) V+\frac{1}{2} \sigma_{1}^{2} X^{2} \partial_{X}^{2} V \\
= & \partial_{t} V+r(X \partial X V-V)+\frac{1}{2} \sigma_{1}^{2} X^{2} \partial_{X}^{2} V \\
& +\sqrt{2} \tilde{\alpha} V \sqrt{1+X \partial X \log V(X \partial X \log V-1)}
\end{aligned}
$$

This is exactly what we obtained in section 2.1 (c.f. Eq. (29)).

\subsection{Relation to Farinelli Connection}

Before concluding this section, we would like to relate our connection $\Gamma$ to another arbitrage connection proposed recently in [Far08], Far09a] and [Far09b]. We will show that the connection presented in [Far08], [Far09a] and [Far09b] is equivalent to Eq. (44). Consider an 
economy in the time interval $t \in[0, T]$, composed of some general "base assets" with prices $X_{i}, i=1, \ldots, N$, and synthetic zero-bonds on these assets. The price of a zero-bond (in the same units as $X_{i}$ ) can be defined as

$$
Z_{i}(t, T):=B_{i}(t, T) X_{i}(t), \quad i=1, \ldots, N
$$

where $B_{i}(T, T)=1$ and $B(t, T)>0$, if $T \geq t$. In other words, $Z_{i}$ pays one unit of asset $X_{i}$ at maturity. Moreover, the dynamics of $B_{i}(t, T)$ can be traced back to a $T$-independent stochastic, which we call the spot rate $r_{i}$. Explicitly,

$$
r_{i}(t):=\lim _{t \rightarrow t^{-}}-\frac{\partial}{\partial T} \log B_{i}(t, T) \text { or equivalently } B_{i}(t, T):=\mathbb{E}_{t}\left[e^{-\int_{t}^{T} r_{i}(s) d s}\right]
$$

The following gauge connection was proposed for this economy 5

$$
\begin{aligned}
F & =\frac{\sum_{i} X_{i}\left(d \phi_{i}-r_{i} \phi_{i} d t\right)}{\sum_{j} \phi_{j} X_{j}} \\
& =d \log \left(\sum_{i} \phi_{i} X_{i}\right)-\frac{\sum_{i} \phi_{i} X_{i}\left(d \log X_{i}+r_{i} d t\right)}{\sum_{j} \phi_{j} X_{j}}
\end{aligned}
$$

Our goal is to relate $F$ to our connection $\Gamma$, Eq. (44), restricted only to the base assets.

In order to do this, we define a larger price vector with components $X_{\mu}, \mu=1, \ldots, 2 N$ :

$$
X(t)=\left(X_{1}(t), \ldots, X_{N}(t), Z_{1}(t, T), \ldots, Z_{N}(t, T)\right)^{\dagger}
$$

We now do the usual decomposition of $d X_{\mu}$ as in Eqs. (2) and (4). Using the pricing formula, Eq. (56), we find that

$$
\begin{aligned}
B_{i}(t, T) & =\mathbb{E}_{t}^{*}\left[e^{-\int_{t}^{T}\left(\alpha^{*}+\sum_{A} \alpha^{A} J_{i+N}^{A}\right) d t^{\prime}+\int_{t}^{T} d \log X_{i}}\right] \\
& =\mathbb{E}_{t}\left[e^{-\int_{t}^{T}\left[\left(\alpha^{*}+\sum_{A} \alpha^{A} J_{i+N}^{A}+\sum_{a}\left(\beta^{a}\right)^{2} / 2\right) d t^{\prime}+\sum_{a} \beta^{a} d W_{a}-d \log X_{i}\right]}\right] \\
& =\mathbb{E}_{t}\left[e^{-\int_{t}^{T} r_{i}(s) d s}\right]
\end{aligned}
$$

where we have extracted the spot rate as

$$
r_{i}=\alpha^{*}+\sum_{A} \alpha^{A} J_{i+N}^{A}+\frac{1}{2} \sum_{a}\left(\beta^{a}\right)^{2}+\sum_{a} \beta^{a} \frac{d W_{a}}{d t}-\frac{d}{d t} \log X_{i} .
$$

Moreover, since $\lim _{T \rightarrow t^{+}} Z_{i}(t, T)=X_{i}(t)$, and by assumption $r_{i}$ is independent of $T$, it must

\footnotetext{
${ }^{5}$ In [Far08, Far09a] and Far09b], Stratonovich calculus was used instead of Itô VKr81]. Here the usual differentiation rules apply.
} 
be that

$$
J_{i+N}^{A}=J_{i}^{A} .
$$

Therefore, we can finally identify the spot rate as:

$$
r_{i}=\alpha^{*}+\sum_{A} \alpha^{A} J_{i}^{A}+\frac{1}{2} \sum_{a}\left(\beta^{a}\right)^{2}+\sum_{a} \beta^{a} \frac{d W_{a}}{d t}-\frac{d}{d t} \log X_{i} .
$$

Inserting Eq. (74) in (69) we obtain

$$
F=-\frac{\sum_{i, A} \alpha^{A} J_{i}^{A} \phi_{i} X_{i}}{\sum_{j} \phi_{j} X_{j}} d t+d \Lambda
$$

where

$$
d \Lambda=-\left(\alpha^{*}+\frac{1}{2} \sum_{a}\left(\beta^{a}\right)^{2}\right) d t-\sum_{a} \beta^{a} d W_{a}+d \log \left(\sum_{i} \phi_{i} X_{i}\right) .
$$

Therefore, we conclude that the connection $F$ is equivalent to $\Gamma$ up to a sign and a gauge transformation.

\section{Measuring Arbitrage Curvature}

In this section we explain how to estimate the arbitrage parameters $\alpha^{A}$ using financial data. Given the discussion in the previous section, measuring these parameters is equivalent to measuring the "curvature" of the market. Needless to say, one can do this for a subset of all instruments only, and there are many technical difficulties which we discuss below.

Even though $\alpha^{A}$ is a gauge invariant, it is still defined up to a rotation in the null space6 $\mathcal{N}$. Therefore, the basic idea is to measure the rotational and gauge invariant quantity

$$
\sum_{\mu, A} \frac{\alpha^{A} J_{\mu}^{A}}{X_{\mu}} \frac{d X_{\mu}}{d t}=\sum_{A}\left(\alpha^{A}\right)^{2} \geq 0
$$

where $\alpha^{A}$ in the left hand side of this equation is expressed as

$$
\alpha^{A}=\sum_{\mu} \frac{J_{\mu}^{A}}{X_{\mu}} \frac{d X_{\mu}}{d t} .
$$

The vectors $J^{A}$ must be calculated using an estimate for the quadratic variation $\Omega_{\mu \nu}:=$

\footnotetext{
${ }^{6}$ For notational simplicity, we will still use $\mathcal{N}$ for the null space of the particular market sub-sector under study. However, it is important to keep in mind that this is not the null space of the full market.
} 
$d\left\langle\log X_{\mu}, \log X_{\nu}\right\rangle / d t$, and the results of Proposition 2.2. We introduce the notation $\mathcal{A}^{2}:=$ $\sum_{A}\left(\alpha^{A}\right)^{2}$ for the measurement of arbitrage curvature. A positive detection of $\mathcal{A}^{2}$ can be translated into a self-financing arbitrage portfolio strategy using the result of the following proposition.

Proposition 4.1 (Arbitrage Strategy): Let the asset corresponding to $\mu=0$ be the numéraire $\left(X_{0}:=1\right)$. If the market model satisfy the positive curvature assumption

$$
\mathcal{A}^{2}>0
$$

then the portfolio allocation

$$
\begin{aligned}
\phi_{0}(t) & :=\sum_{i=1}^{N} \int_{0}^{t} \phi_{i}(s) d X_{i}(s)+\sum_{A} J_{0}^{A}(t) \alpha^{A}(t), \\
\phi_{i}(t) & :=\sum_{A} \frac{J_{i}^{A}(t) \alpha^{A}(t)}{X_{i}(t)} \quad(i=1, \ldots, N),
\end{aligned}
$$

is a selfinancing arbitrage strategy delivering wealth

$$
V(t)=\int_{0}^{t} \mathcal{A}^{2}(s) d s
$$

Proof: First, we check that the strategy is self-financing, that is

$$
\sum_{\mu=0}^{N}\left(d \phi_{\mu} X_{\mu}+d\left\langle\phi_{\mu}, X_{\mu}\right\rangle\right)=0
$$

where $d$ denotes the Itô differential (see [LaLa06], Chapter 4.1.2). This is proved by the 
following computation:

$$
\begin{aligned}
\sum_{\mu=0}^{N}\left(d \phi_{\mu} X_{\mu}+d\left\langle\phi_{\mu}, X_{\mu}\right\rangle\right)= & d \phi_{0} X_{0}+d\left\langle\phi_{0}, X_{0}\right\rangle+\sum_{i=1}^{N}\left(d \phi_{i} X_{i}+d\left\langle\phi_{i}, X_{i}\right\rangle\right) \\
= & \sum_{A} \sum_{i=1}^{N} \alpha^{A} J_{i}^{A} d X_{i} / X_{i}+\sum_{A} d\left(\alpha^{A} J_{0}^{A}\right) \\
& +\sum_{i=1}^{N} X_{i} d\left(\sum_{A} \alpha^{A} J_{i}^{A} / X_{i}\right)+\sum_{i=1}^{N} d\left\langle\sum_{A} \alpha^{A} J_{i}^{A} / X_{i}, X_{i}\right\rangle \\
= & \sum_{A} d\left(\alpha^{A} \sum_{\mu=0}^{N} J_{\mu}^{A}\right) \\
= & 0 .
\end{aligned}
$$

Since the self-financing condition is fulfilled, the portfolio value can be computed as

$$
\begin{aligned}
V(t) & =\sum_{\mu=0}^{N} \phi_{\mu}(t) X_{\mu}(t)=\int_{0}^{t} \phi_{i}(s) d X_{i}(s)+\sum_{A} \alpha^{A} \underbrace{\sum_{\mu=0}^{N} J_{\mu}^{A}}_{=0} \\
& =\int_{0}^{t} \sum_{A} \alpha^{A}(s) \sum_{i=1}^{N} \frac{J_{i}^{A}(s)}{X_{i}(s)} d X_{i}(s)=\int_{0}^{t} \sum_{A} \alpha^{A}(s) \sum_{\mu=0}^{N} \frac{J_{\mu}^{A}(s)}{X_{\mu}(s)} d X_{\mu}(s) \\
& =\int_{0}^{t} \sum_{A} \alpha^{A}(s)^{2} d s=\int_{0}^{t} \mathcal{A}^{2}(s) d s .
\end{aligned}
$$

Since the arbitrage curvature are positive, we see that $V(0)=0$ and $V(t)>0$ for all times $t \in[0, T]$. The proof is completed.

Of course, there is no continuous time trading in the markets, and we can only do measurements in discrete time. Moreover, our estimate of $\Omega$ will always include errors. This means that we will always have a noise term in the right hand side of Eq. (77)). The goal of this section is to explain the basic steps used to measure arbitrage curvature, and understand the major sources of error in such measurements. A key aspect of our algorithm is that we test directly for the gauge invariance of the arbitrage signal. This allow us to check the robustness of our estimators. We find that the gauge invariance of the arbitrage signal, as predicted by the stochastic models, is indeed obeyed with good accuracy in the real market. 


\subsection{Basic Algorithm}

In what follows, we will use a hat in any variable which is an estimate of some parameter, e.g. $\hat{\Omega}$ is an estimate for $\Omega$. The first problem we face is to find an estimate for the quadratic variation $\Omega$ and to determine the null space $\mathcal{N}$ defined in section 2 (if non-trivial). This is a familiar problem in volatility modeling. Since we will never observe $\Omega$ directly, it is expected that our estimate will not have any exact zero-mode, but only eigenvectors with small eigenvalues. In fact, a priori, we do not know if the space $\mathcal{N}$ is non-trivial. We can only guess its dimension.

Let $\hat{\Omega}$ be any estimate for $\Omega$. Then, following Proposition 2.2, we construct the matrix

$$
\hat{G}=\hat{\Omega}-\frac{1}{N}(U \hat{\Omega}+\hat{\Omega} U)+\frac{1}{N^{2}} \operatorname{Tr}(U \hat{\Omega}) U
$$

where $N$ is the number of rows (or columns) of $\Omega$ and $U$ is the matrix of all ones: $U_{\mu \nu}=$ $1, \forall \mu, \nu$. We can then use standard algorithms to compute the eigenspace of $\hat{G}$. This will yield orthonormal eigenvectors

$$
\hat{G} \hat{J}^{A}=\lambda^{A} \hat{J}^{A}, \quad\left(\hat{J}^{A}\right)^{\dagger} \hat{J}^{B}=\delta^{A B}, \quad A, B=0,1, \ldots, N-1,
$$

where $\lambda^{A} \geq 0$ since $\hat{G}$ is positive semidefinite. As a matter of fact, since $\Omega$ and $U$ commute, the have a common basis of eigenvectors and a short computation proves that $G$ has always (at least) one zero eigenvalue and the biggest $N-1$ eigenvalues equal those of $\Omega$, which are not negative (c.f. Proposition 2,2). In practice, there will only be one exact zero eigenvector: $\hat{J}^{0} \propto(1,1, \ldots, 1)^{\dagger}$. Summarizing, the eigenvalues of $G$ ordered in increasing order of magnitude are,

$$
0=\lambda^{0} \leq \lambda^{1} \leq \lambda^{2} \leq \ldots \leq \lambda^{N-1}
$$

It is easy to show (c.f. Proposition 2.2) that

$$
\sum_{\mu} \hat{J}_{\mu}^{A}=0, \quad \text { for } A=1,2, \ldots, N-1 .
$$

Our estimate for the basis of $\mathcal{N}$ will be to chose the first $k$ eigenvectors with the smallest eigenvalues: $\hat{J}^{A}, A=1, \ldots, k<N-1$. In doing this, we are assuming that $\operatorname{dim}(\mathcal{N})=k$.

Once, we have calculated $\hat{J}^{A}$, we can compute our estimate of $\alpha^{A}$ in discrete time:

$$
\hat{\alpha}^{A}(t+\delta t)=\sum_{\mu} \frac{\hat{J}_{\mu}^{A}(t)}{\delta t X_{\mu}(t)}\left[X_{\mu}(t+\delta t)-X_{\mu}(t)\right] .
$$


Note that $\hat{J}^{A}(t)$ is constructed with information up to time $t$ only. This estimate is consistent with the non-anticipating nature of Itô integrals. The time step $\delta t$ is, of course, arbitrary. Our estimate for $\mathcal{A}^{2}$ now becomes:

$$
\hat{\mathcal{A}}^{2}(t+\delta t)=\sum_{A=1}^{k}\left[\hat{\alpha}^{A}(t)\right]^{2}+\sum_{A=1}^{k} \hat{\alpha}^{A}(t)\left[\hat{\alpha}^{A}(t+\delta t)-\hat{\alpha}^{A}(t)\right]
$$

In the limit of short time scales, and if there is non-trivial arbitrage, we expect that this estimator converges to the true signal

$$
\hat{\mathcal{A}}^{2}(t+\delta t)=\mathcal{A}^{2}(t)+\sum_{A} \alpha^{A} d \alpha^{A}=\mathcal{A}^{2}(t)+\mathcal{O}(\delta t) \quad(\delta t \rightarrow 0) .
$$

The convergence in Eq. (91) is only valid if in the limit $\delta t \rightarrow 0$ we have

$$
\mathbb{E}_{t}\left[\hat{\alpha}^{A}(t+\delta t)\right]-\hat{\alpha}^{A}(t)=\mathcal{O}(\delta t), \quad \operatorname{Cov}_{t}\left[\hat{\alpha}^{A}(t+\delta t), \hat{\alpha}^{B}(t+\delta t)\right]=\mathcal{O}(\delta t)
$$

Therefore, we expect that, if there is non-trivial arbitrage in the market, the estimator (90) will give us a positive signal on average. Since the time scale is arbitrary, it is convenient to set $\delta t=1$ henceforth.

There are several candidates for an estimator for $\Omega$. The "right" choice of $\hat{\Omega}$ should reflect our believes about the true dynamics of the asset values. Here we will simply take the empirical estimator for covariance of the time series of log returns for a window of length $L$. More precisely, our data consist of a number of time series for the prices $X_{\mu}, \mu=0, \ldots, N-1$ in certain units, say USD7. Our estimator reads

$$
\begin{aligned}
\hat{\Omega}_{\mu \nu}(t)= & \frac{1}{L} \sum_{i=0}^{L-1} \log \left[\frac{X_{\mu}(t-i)}{X_{\mu}(t-i-1)}\right] \log \left[\frac{X_{\nu}(t-i)}{X_{\nu}(t-i-1)}\right] \\
& -\frac{1}{L^{2}} \sum_{i, j=0}^{L-1} \log \left[\frac{X_{\mu}(t-i)}{X_{\mu}(t-i-1)}\right] \log \left[\frac{X_{\nu}(t-j)}{X_{\nu}(t-j-1)}\right] .
\end{aligned}
$$

For more sophisticated estimators see [HaHaPi08, Zh06]. We are now in position to summarize the most basic algorithm to detect arbitrage.

\section{Algorithm:}

1. Starting with the time series for $X_{\mu}, \mu=0, \ldots, N-1$ in an interval $[t, t-L]$, we estimate $\hat{\Omega}_{\mu \nu}(t)$ using Eq. (93).

\footnotetext{
${ }^{7}$ We also include the USD itself as an asset in which we have $X_{0}=1$.
} 
2. We then calculate the $\hat{G}$ matrix using Eq. (85), and its orthonormal eigenspace. The eigenvectors will be labeled as $\hat{J}^{A}, A=0,1, \ldots, N-1$, in order of increasing eigenvalues: $0=\lambda^{0} \leq \lambda^{1} \leq \ldots \leq \lambda^{N-1}$. Moreover, $\hat{J}^{0} \propto(1,1, \ldots, 1)^{\dagger}$.

3. Given a guess for the dimension of the null space $k=\operatorname{dim}(\mathcal{N})$, we take as its basis the following eigenvectors of $\hat{G}: \hat{J}^{A}, A=1, \ldots, k$.

4. We then calculate $\hat{\alpha}^{A}(t+1)$ from Eq. (89), which uses information up to time $t+1$.

5. Roll the time window by one step, and repeat steps 1-5. Once we have more than one estimate for $\hat{\alpha}^{A}$, we can calculate our final arbitrage estimator $\hat{\mathcal{A}}^{2}$ from Eq. (90).

6. In order to explicitly check for gauge invariance, we repeat steps 1-5, using each asset $X_{\mu}$ as numeraire. For example, if we want to use $X_{1}$ as numeraire, we divide all elements of the time series by the corresponding element of $X_{1}$, e.g. $X_{\mu}(s) \rightarrow X_{\mu}(s) / X_{1}(s)$ $\forall \mu$ and $s \in[t, t-L]$. Then we repeat steps $1-5$ with the new time series. Note that this is a non-trivial transformation in the data and, in practice, we will get different estimates for $\hat{\alpha}^{A}$.

Before discussing the results of the algorithm, we need to understand what are the main sources of error in our signal. This is done in the next subsection.

\subsection{Sources of Error}

The sources of error in our measurement of $\mathcal{A}^{2}$ can be divided in three groups. First, there is gauge dependence. Second, there is a gauge invariant noise, which we will discuss below. Finally, when using high-frequency financial data, one is faced with the so-called market microstructure noise which is partly due to the bid/ask bounce effect [HaHaPi08].

We begin with looking at sources of gauge dependence. Note that our construction of the estimators assumes that, under a gauge transformation, $\hat{\Omega}$ transforms like $\Omega$, c.f. Eq. (14). However, the gauge transformation rule in the real world can be quite different, because the unknown effective dynamics could lead to gauge dependences. We do not have an a priori test for this source of error. The only way to test for it is to make our calculations in different gauges and see how different the answers are. We will show examples of this in the next sections.

The second source of error in our signals come from a gauge invariant noise term. In fact, we will see that this is the dominant noise contribution. In order to understand this noise, 
it is convenient to discretize the Itô integral and write our estimate for $\alpha^{A}$ as

$$
\begin{aligned}
\hat{\alpha}^{A}(t+1)= & \sum_{\mu} \frac{\hat{J}_{\mu}^{A}(t)}{X_{\mu}(t)}\left[X_{\mu}(t+1)-X_{\mu}(t)\right] \\
= & \sum_{\mu, B} \hat{J}_{\mu}^{A}(t) J_{\mu}^{B}(t) \alpha^{B}(t)+\sum_{\mu, a} \hat{J}_{\mu}^{A}(t) \sigma_{\mu}^{a} \beta^{a}(t) \\
& +\sum_{\mu, a} \hat{J}_{\mu}^{A}(t) \sigma_{\mu}^{a}\left[W_{a}(t+1)-W_{a}(t)\right] \\
:= & \alpha_{\text {trend }}^{A}(t)+\epsilon^{A}(t+1) .
\end{aligned}
$$

Here we have decomposed the signal in a trend

$$
\alpha_{\text {trend }}^{A}(t):=\sum_{\mu, B} \hat{J}_{\mu}^{A}(t) J_{\mu}^{B}(t) \alpha^{B}(t)+\sum_{\mu, a} \hat{J}_{\mu}^{A}(t) \sigma_{\mu}^{a} \beta^{a}(t)
$$

and a stochastic noise term

$$
\epsilon^{A}(t+1):=\sum_{\mu, a} \hat{J}_{\mu}^{A}(t) \sigma_{\mu}^{a}\left[W_{a}(t+1)-W_{a}(t)\right]
$$

with $\mathbb{E}_{t}\left[\epsilon^{A}(t+1)\right]=0$. Since $\hat{J}^{A}$ is only an estimate for the real $J^{A}$, we have that $\sum_{\mu} \hat{J}^{A} \sigma_{\mu}^{a} \neq 0$ in general. Therefore, our error in the estimate of $J^{A}$ will induce an extra noise term in the signal. Moreover, it will also induce some gauge dependency. To see this, note that under a change of numéraire, we have $\sigma_{\mu}^{a} \rightarrow \sigma_{\mu}^{a}+\delta \sigma^{a}$ and $\beta^{a} \rightarrow \beta^{a}+\delta \sigma^{a}$. It is then easy to check that the trend will transform as $\alpha_{\text {trend }}^{A} \rightarrow \alpha_{\text {trend }}^{A}+\sum_{\mu, a} \hat{J}_{\mu}^{A} \sigma_{\mu}^{a} \delta \sigma^{a}$. However, note that the noise term is gauge invariant. In fact, one expects the term $\sum_{\mu} \hat{J}_{\mu}^{A} \sigma_{\mu}^{a}$ to be quite small. Moreover, since in our algorithm the gauge transformation is of the order $\delta \sigma^{a}=\mathcal{O}\left(\sigma_{\mu}^{a}\right)$, we expect the gauge dependence coming from the trend to be negligible. We will see that, in real financial data, most of the signal can be accounted by the gauge invariant noise term.

We are interested in estimating the size of the noise contribution. For that, we compute 
the variance of the noise using information up to time $t$ :

$$
\begin{aligned}
\operatorname{Var}_{t}\left[\sum_{A} \hat{\alpha}^{A}(t) \hat{\alpha}^{A}(t+1)\right]= & \mathbb{E}_{t}\left\{\left[\sum_{A} \hat{\alpha}^{A}(t)\left(\hat{\alpha}^{A}(t+1)-\mathbb{E}_{t}\left[\hat{\alpha}^{A}(t+1)\right]\right)\right]^{2}\right\} \\
= & \sum_{A, B} \hat{\alpha}^{A}(t) \hat{\alpha}^{B}(t)\left(\left[\hat{J}^{A}(t)\right]^{\dagger} G(t) \hat{J}^{B}(t)\right) \\
= & \sum_{A}\left[\hat{\alpha}^{A}(t)\right]^{2} \lambda^{A}(t) \\
& +\sum_{A, B} \hat{\alpha}^{A}(t) \hat{\alpha}^{B}(t)\left(\left[\hat{J}^{A}(t)\right]^{\dagger} \delta G(t) \hat{J}^{B}(t)\right)
\end{aligned}
$$

where $\delta G=G-\hat{G}$. If we think that our estimate of $G$ is good, we can neglect the $\mathcal{O}(\delta G)$ term and approximate

$$
\operatorname{Var}_{t}\left[\hat{\mathcal{A}}^{2}(t+1)\right] \approx \sum_{A}\left[\hat{\alpha}^{A}(t)\right]^{2} \lambda^{A}(t) \leq \sum_{A}\left[\hat{\alpha}^{A}(t)\right]^{2} \lambda_{k}(t)
$$

where we remind the reader that $k$ is our estimate for the dimension of $\mathcal{N}$, and the eigenvalues of $\hat{G}$ have been ordered so that $\lambda^{1} \leq \lambda^{2} \leq \ldots \leq \lambda^{k}$.

An interesting consequence of Eq. (98) is that one can put a fundamental bound for the size of the arbitrage curvature, in order to be detectable. We have,

$$
\mathcal{A}^{2} \geq \sqrt{\operatorname{Var}_{t}\left[\hat{\mathcal{A}}^{2}\right]} \Longrightarrow \mathcal{A}^{2} \geq \lambda_{k}
$$

This means that, in order to have a chance to detect arbitrage, one needs to find financial products whose time series are as correlated as possible, which implies a very small value of $\lambda_{k}$.

The third source of error is market microstructure noise. This effect is relevant in high frequency data, when the size of the price movements is comparable with the bid/ask spread. In order to model this noise, it is convenient to set $X_{0}:=1$ as our numéraire. The standard way of simulating this noise is to introduce an additional jump term $\eta_{i}(t)$ to the log prices $X_{i}, i=1, \ldots, N$. More precisely, the observed price is $\tilde{X}_{i}$ and it is given by,

$$
\log \tilde{X}_{i}(t)=\log X_{i}(t)+\eta_{i}(t)
$$

where $X_{i}$ is the "true" Itô process, and for simplicity we assume

$$
\mathbb{E}\left[X_{i} \eta_{j}\right]=0, \quad \mathbb{E}\left[\eta_{i}\right]=0, \quad \mathbb{E}\left[\eta_{i} \eta_{j}\right]:=\eta^{2} \delta_{i j}
$$


Moreover, the noise terms are uncorrelated between different times. One can then show that our estimator will be contaminated by an amount

$$
\lim _{\delta t \rightarrow 0} \mathbb{E}\left[\hat{\mathcal{A}}^{2}\right]=\mathbb{E}\left[\mathcal{A}^{2}\right]-\gamma \frac{\eta^{2}}{\delta t^{2}}+\mathcal{O}\left(\frac{\eta^{4}}{\delta t^{2}}\right),
$$

where $\gamma:=\operatorname{dim}(\mathcal{N})-\mathbb{E}\left[\left(\sum_{A} J_{0}^{A}\right)^{2}\right]$. It can be shown that $\gamma \geq 0$. Therefore, we see that the microstructure noise leads a negative contribution to our estimation of $\mathcal{A}^{2}$. The absolute value of such contribution diverges as we move towards higher frequencies $(\delta t \rightarrow 0)$.

One way of detecting the presence of microstructure noise is to note that

$$
\lim _{\delta t \rightarrow 0} \mathbb{E}\left[\log \left(\tilde{X}_{i}(t+\delta t) / \tilde{X}_{i}(t)\right) \log \left(\tilde{X}_{i}(t) / \tilde{X}_{i}(t-\delta t)\right)\right]=-\eta^{2}<0
$$

In other words, the microstructure noise induces a negative correlation between subsequent $\log$ returns. We find that this effect is quite pronounced for equity and futures data. However, for stock indices, the effect seems to be negligible. This is mainly due to the fact that the microstructure noise "averages out" between all the stocks in the index.

There is an extra source of error which is intrinsic to the algorithm, but only if we use a rolling window in our estimation of $\hat{J}^{A}$. For example, suppose that we estimate $\hat{J}^{A}(t)$ and then roll the window and estimate $\hat{J}^{A}(t+1)$. Even if the matrices $\hat{G}(t)$ and $\hat{G}(t+1)$ are near, $\hat{J}^{A}(t)$ and $\hat{J}^{A}(t+1)$ can differ by a large orthogonal transform. It can be just a sign flip f.i., since the eigenvalue equations are invariant under $\hat{J}^{A} \rightarrow-\hat{J}^{A}$. However, suppose two eigenvalues are near to each other, i.e. $\lambda_{1} \approx \lambda_{2}$. Then, any linear combination of $\hat{J}^{1}$ and $\hat{J}^{2}$ is also approximatively an eigenvector of $\hat{G}$. In physics, this is known as the problem of degenerate perturbation theory (see f.i. [Sa94]). More generally, we have that

$$
\lim _{\|\hat{G}(t)-\hat{G}(t+1)\| \rightarrow 0} \hat{J}^{A}(t+1)=\sum_{B} C^{A B} \hat{J}^{B}(t),
$$

where $C$ is an orthogonal matrix, i.e.

$$
C^{\dagger} C=1
$$

The problem can be solved if we can determine $C$. If so, we can construct the "correct" eigenvectors $\tilde{J}^{A}(t+1):=\sum_{B}\left(C^{\dagger}\right)^{A B} \hat{J}^{B}(t+1)$ so that $\lim _{\|\delta \hat{G}\| \rightarrow 0} \tilde{J}^{A}(t+1)=\hat{J}^{A}(t)$. An approximate solution for $C$ is presented in appendix A. This is implemented in our numerical routines. 


\subsection{Simulated Data}

In this section we apply our algorithm to simulated financial data. For this, we study the simple log-normal random walk model with constant coefficients (c.f. Eq. (2)). The solution to the stochastic differential equation (2) is

$$
X_{\mu}(t)=X_{\mu}(0) \exp \left[\left(\alpha_{\mu}-\frac{1}{2} \sum_{a=1}^{d}\left(\sigma_{\mu}^{a}\right)^{2}\right) t+\sum_{a=1}^{d} \sigma_{\mu}^{a} B_{a}(t)\right]
$$

where $B(t):=\left[B_{1}(t), \ldots, B_{d}(t)\right]^{\dagger}$ is standard multivariate Brownian motion with

$$
\mathrm{E}\left[B_{a}(t)\right]=0, \quad \operatorname{Cov}\left[B_{a}(t), B_{b}(t)\right]=t \delta_{a b},
$$

for all $a, b=1, \ldots, d$. As usual, we decompose the trends as

$$
\alpha_{\mu}=\alpha+\sum_{a=1}^{d} \beta^{a} \hat{\sigma}_{\mu}^{a}+\sum_{A \in \mathcal{N}} \alpha^{A} J_{\mu}^{A}, \quad \hat{\sigma}_{\mu}^{a}=\sigma_{\mu}^{a}-\frac{1}{N} \sum_{\nu=0}^{N-1} \sigma_{\nu}^{a} .
$$

We begin with an example with $N=21$ assets and $d=18$ Brownian motions, which implies $k=\operatorname{dim}(\mathcal{N})=2$. We take as a first asset a bank account with zero interest rate, and make it to our numéraire. This means that we choose,

$$
X_{0}: \equiv 1, \quad \sigma_{0}^{a}=0, \quad \alpha_{0}=0
$$

which implies

$$
\alpha=\frac{1}{N} \sum_{i=1}^{N-1} \sum_{a=1}^{d} \beta^{a} \sigma_{i}^{a}-\sum_{i=1}^{N-1} \sum_{A=1}^{2} \alpha^{A} J_{i}^{A} .
$$

In figure 1 we show a particular simulation of the $\log$ prices, where we take $\beta^{a}, \sigma_{i}^{a}, \alpha^{A}$ from uniform random distributions in the intervals, $\beta^{a} \in\left[-10^{-4}, 10^{-4}\right], \sigma_{i}^{a} \in\left[-10^{-3}, 10^{-3}\right]$ and $\alpha^{A} \in\left[-10^{-4}, 10^{-4}\right]$. The simulation was generated using Mathematica. The arbitrage detection algorithm was implemented in $\mathrm{C}++$. Each price was taken at a time separation of $\Delta t=1$ (arbitrary time unit). In this particular case we calculate $\hat{\Omega}$ using the first 100 prices of the time series. In other words, we do not use a moving window. The results with the moving window are very similar.

Now suppose we assume (correctly) that we have $k=\operatorname{dim}(\mathcal{N})=2$. We then run the algorithm and find the signal shown in figure 2 . The solid horizontal line at $\mathcal{A}^{2} \approx 10^{-8}$ is the correct value of $\mathcal{A}^{2}$. Therefore, we see that we get an accurate estimate for the arbitrage curvature. Note that, as we discussed in the previous section, in our algorithm we compute $\hat{\mathcal{A}}^{2}$ using each of the different assets as numéraire. We include error bars showing the range 


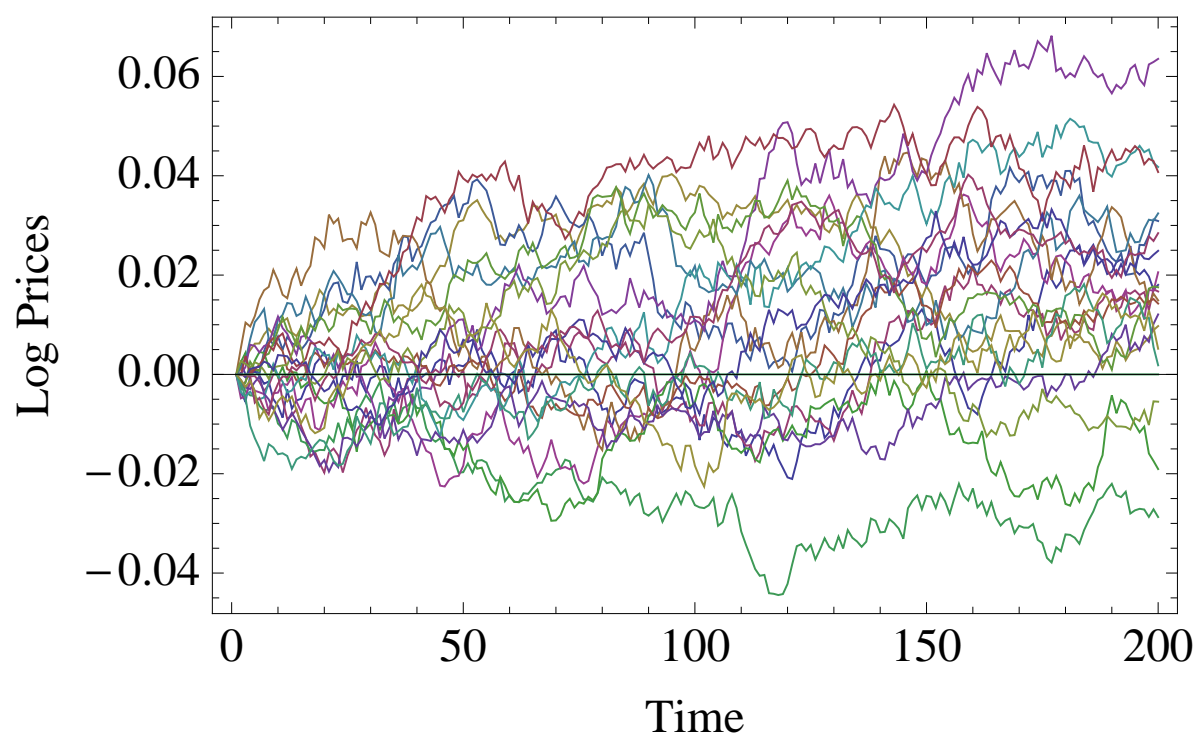

Figure 1: Simulation with 20 log-normal random walks.

of values obtained using the different gauges. The results in this simulated sample are gauge invariant to such high accuracy that the error bars cannot be appreciated.

In the previous section we discussed how the main source of error in our detection technique can be related to the biggest eigenvalue $\lambda_{k}$ of the set $\left\{\lambda_{1}, \ldots, \lambda_{k}\right\}$. This led to a gauge invariant noise term. In this simulated sample data, we find that $\lambda_{k} \approx 10^{-21}$, and so using Eq. (98) we find, $\sqrt{\operatorname{Var}\left[\hat{\mathcal{A}}^{2}\right]} \approx \sqrt{10^{-8} \times 10^{-21}} \approx 10^{-15}$. Therefore, this noise term is very small in this case. The fluctuations seen in figure 2 are an artifact of this particular model. To understand them, we can expand Eq. (106) as

$$
\frac{X_{\mu}(t+1)-X_{\mu}(t)}{X_{\mu}(t)}=\alpha_{\mu}+\sum_{a} \sigma_{\mu}^{a} B_{a}(1)+\epsilon_{\mu}+\ldots
$$

where

$$
\epsilon_{\mu}=\frac{1}{2}\left(-\Omega_{\mu \mu}+\sum_{a, b} \sigma_{\mu}^{a} \sigma_{\mu}^{b} B_{a}(1) B_{b}(1)\right) .
$$

It is easy to show that $\epsilon_{\mu}$ is gauge invariant and

$$
\mathbb{E}\left[\epsilon_{\mu}\right]=0, \quad \mathbb{E}\left[\epsilon_{\mu} \epsilon_{\nu}\right]=\frac{1}{2}\left(\Omega_{\mu \nu}\right)^{2}
$$

This extra noise term, $\epsilon_{\mu}$, is the reason for the gauge invariant fluctuations in figure 2 . The noise term vanishes, if we integrate $d X_{\mu}$ using an infinite partition of the time interval, as it is assumed in Itô integrals. Of course, this is never possible in practice. Nevertheless, we see 
that in this example, the extra noise is very small compared to the arbitrage parameter $\mathcal{A}^{2}$. In fact, we expect this noise to be very small in general since it is of order $\operatorname{Var}\left[\epsilon_{\mu}\right]=O\left(\left(\sigma_{\mu}^{a}\right)^{4}\right)$.

It is interesting to see what happens if we assume the wrong number of zero modes. For example, in figure 3 we show what happens if we take $k=1$. We see that we get a gauge dependent signal. Finally, in figure 4 we show what happens if we assume $k=3$. In this case, the biggest eigenvalue is $\lambda_{k} \approx 10^{-8}$. As the figure shows, most of the fluctuations are coming from the gauge invariant noise described in the previous section. To see this we have plotted the expected noise according to Eq. (98):

$$
\operatorname{noise}_{ \pm}(t+1)=\left(\mathcal{A}^{2} \pm \sqrt{\operatorname{Var}_{t}\left[\hat{\mathcal{A}}^{2}(t+1)\right]}\right)=\left(\mathcal{A}^{2} \pm \sqrt{\sum_{A=1}^{k}\left(\hat{\alpha}^{A}(t)\right)^{2} \lambda^{A}(t)}\right)
$$

where $\mathcal{A}^{2} \approx 10^{-8}$ is the true value of the arbitrage (which is also the mean of the signal). We see that this noise accounts for most of the fluctuations and it makes the true arbitrage signal almost undetectable. The main point we would like to make here is that the correct value of $k$ can be estimated from the quality of the signal.

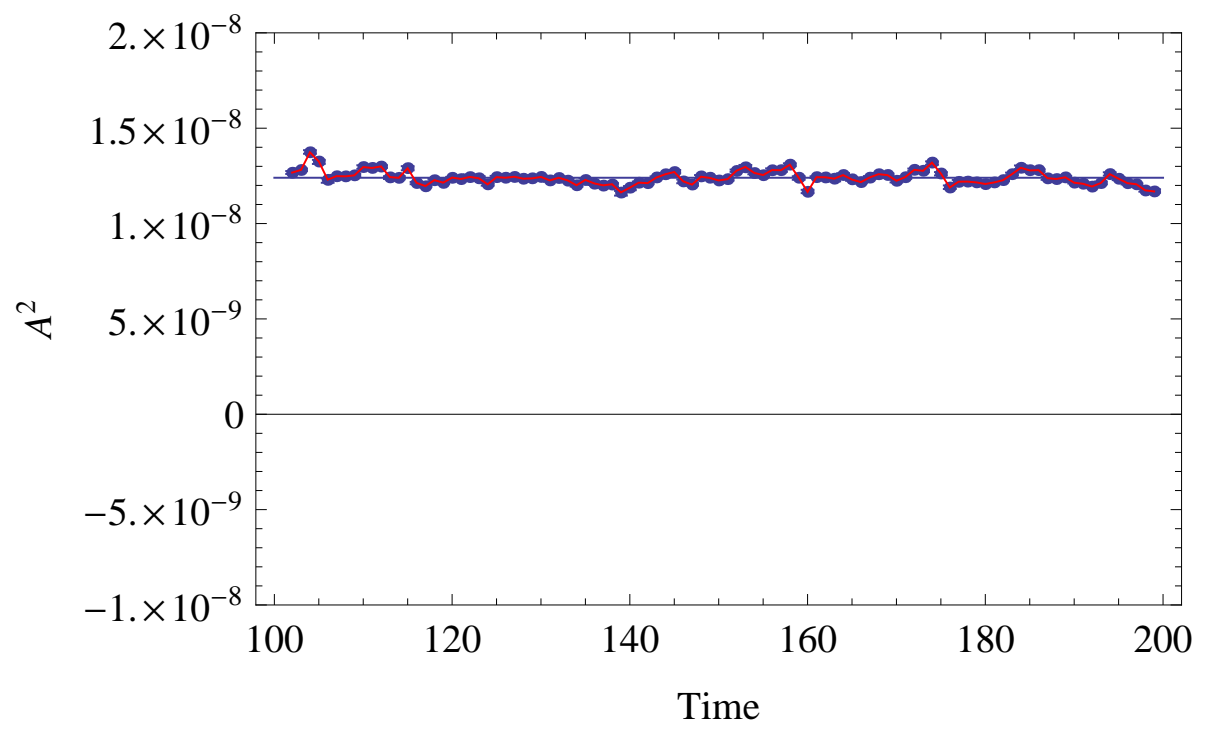

Figure 2: Result for the arbitrage detection algorithm applied to the simulated data in figure 1. Here we assume (correctly) $k=2$ null directions. The solid horizontal line at $\mathcal{A}^{2} \sim 10^{-8}$ is the correct value of $\mathcal{A}^{2}$. The red solid line is the USD value of the signal.

We can now investigate the effect of the market microstructure noise discussed in the previous section. In order to do this, we include additional white noise terms to the price processes of Eq. (106) as described in the previous sub-section (c.f. Eq. (100)). In this particular example we choose the variance to be $\eta^{2}=10^{-5}$. We then apply the noise to 


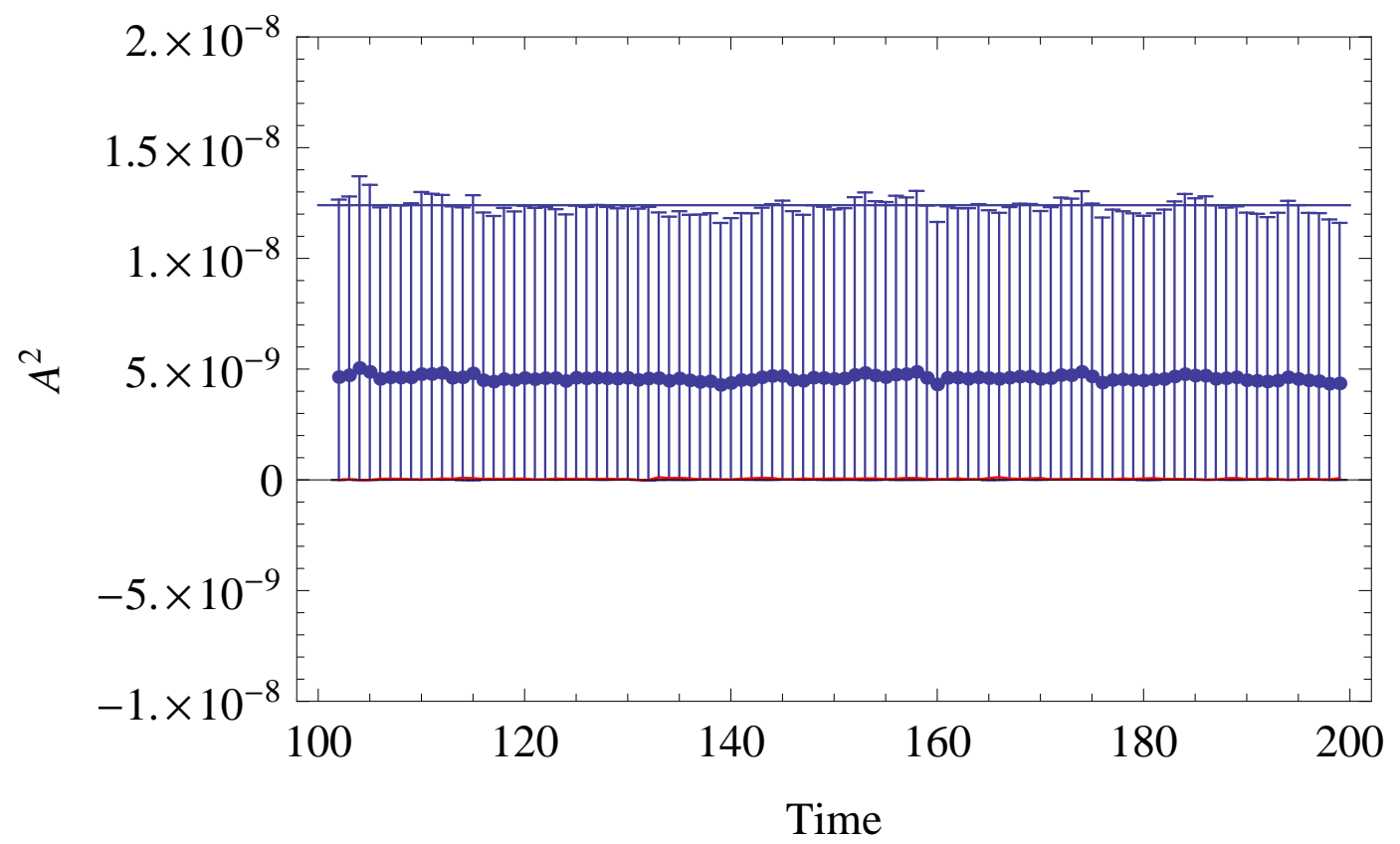

Figure 3: Result for the arbitrage detection algorithm applied to the simulated data in figure 1. Assuming $k=1$ null directions (incorrectly), and using a fixed window of 100 time steps. The error bars give the range of values obtained using the different gauges. The solid dots are the mean of all results. The red line is the USD signal.

the data of the previous example. In figure 4 we show the result of the estimate of $\mathcal{A}^{2}$ for the contaminated data. In this case we assume (correctly) that the dimension of the null space is $k=2$. We can clearly see how the signal is now negative on average, due to the microstructure noise. This matches the theoretical prediction in Eq. (102). In figure 6 we plot the product of subsequent log returns according to (c.f. Eq. (103))

$$
\hat{\eta}^{2}(t):=-\frac{1}{N-1} \sum_{i=1}^{N-1} \log \left(\tilde{X}_{i}(t+1) / \tilde{X}_{i}(t)\right) \log \left(\tilde{X}_{i}(t) / \tilde{X}_{i}(t-1)\right)
$$

where $\tilde{X}_{i}$ are the contaminated prices. According to Eq. (103) of the previous sub-section, we should have $\mathbb{E}\left[\hat{\eta}^{2}\right]=\eta^{2}$. This is precisely what we observe in figure 6 . In the next subsection, we will see that such signals are very typical of high frequency security prices.

\subsection{Market Data}

In this section we present some examples of our arbitrage detection algorithm applied to real financial data. We begin with a look at three major US stock indexes: the Dow Jones Composite Average (DJA), the NASDAQ Composite Index (IXIC) and the NYSE Composite 


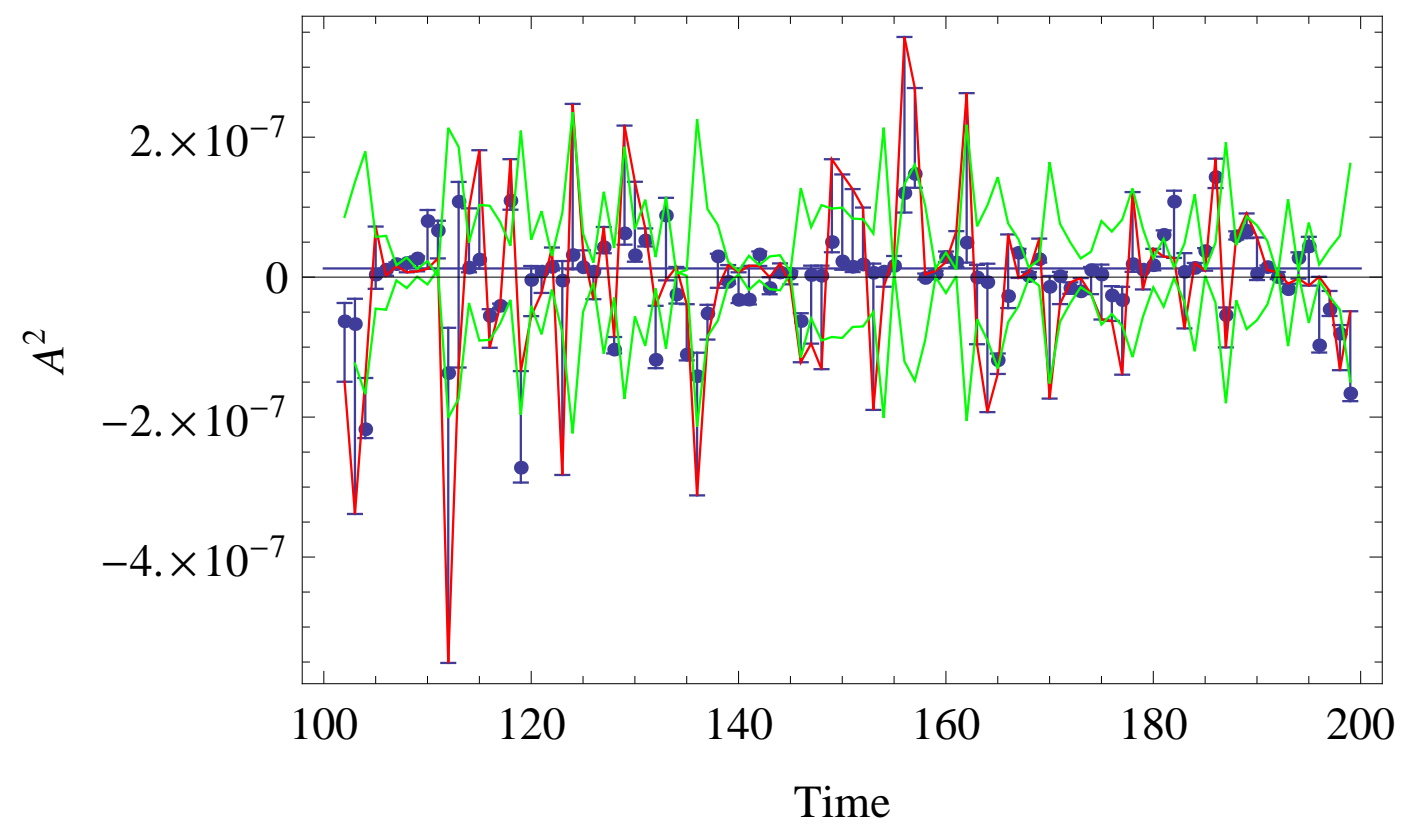

Figure 4: Result for the arbitrage detection algorithm applied to the simulated data in figure 1. Assuming $k=3$ null directions (incorrectly), and using a fixed window of 100 time steps. The green lines are the noise terms noise $e_{ \pm}$estimated according to Eq. (114). The error bars give the range of values obtained using the different gauges. The solid dots are the mean of all results. The red line is the USD signal.

Index (NYA). Due to their similar nature, we expect strong correlations between these indexes. Our first sample consist of daily closing prices from September 12004 to July 16 2009, a total of 1227 data points. The gauge invariant matrix $\hat{G}$ has been estimated using a moving window of 500 days. We have found the following values for the eigenvalues: $\lambda_{1} \approx 2 \times 10^{-3}, \lambda_{2} \approx 5 \times 10^{-3}, \lambda_{3} \approx 2 \times 10^{-2}$. Therefore, it is reasonable to assume that the null space has only one dimension, $k=1$. The result of the arbitrage detection algorithm is shown in figure 7 . We can see that the signal is indeed gauge invariant to a very good accuracy. In figure 7 we have also included an estimate for the gauge invariant noise term described in section 4.2. In this case we have assumed that the average of the signal is zero (i.e. no-arbitrage), and so our estimate for the expected noise is

$$
\operatorname{noise}_{ \pm}(t+1)= \pm \sqrt{\operatorname{Var}_{t}\left[\hat{\mathcal{A}}^{2}(t+1)\right]}= \pm \sqrt{\sum_{A=1}^{k}\left(\hat{\alpha}^{A}(t)\right)^{2} \lambda_{A}(t)}
$$

Looking at figure 7 , we see that the noise can explain most of the signal. Therefore, we find that our results are consistent with $\mathcal{A}^{2}=0$, and hence no arbitrage. In figure 8 we show a histogram of the different values of $\hat{\mathcal{A}}^{2}$. As pointed out above, the signal is consistent with 


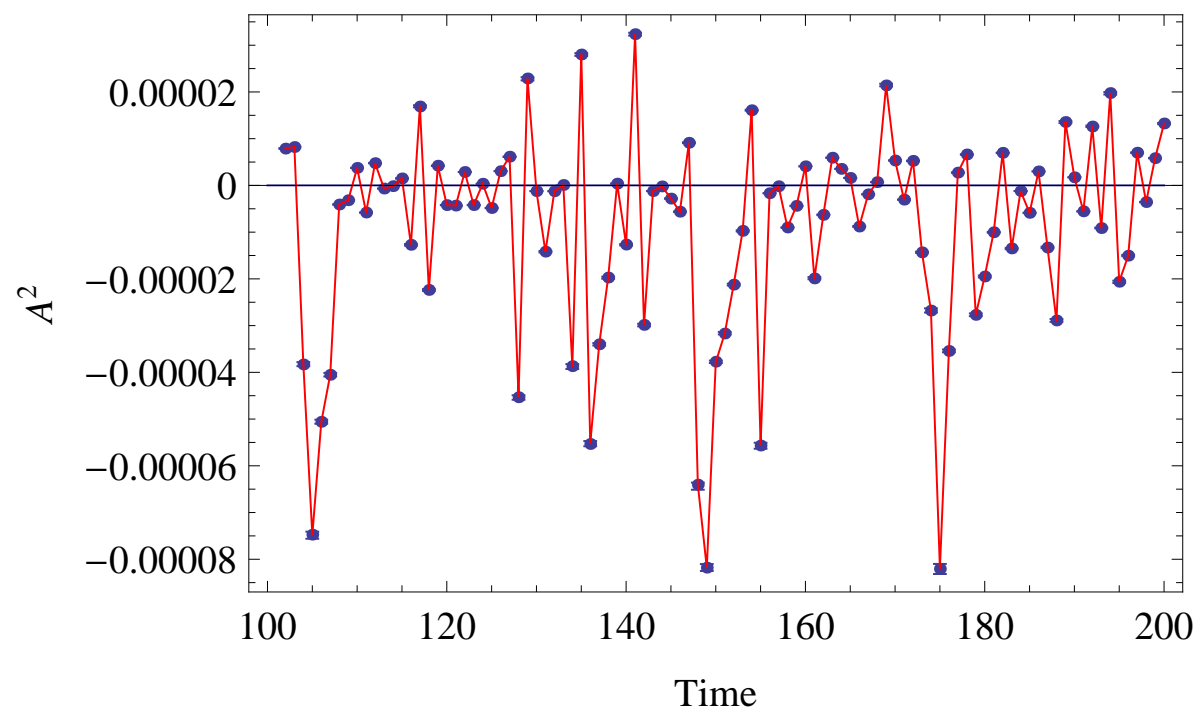

Figure 5: Result for the arbitrage detection algorithm applied to the simulated data in figure 1, now including microstructure noise with variance $\eta^{2}=10^{-5}$. Here we assume (correctly) $k=2$ null directions.

$\mathcal{A}^{2}=0$ since the signal to noise ratio is very low: $\mathbb{E}\left[\hat{\mathcal{A}}^{2}\right] / \sqrt{\operatorname{Var}\left[\hat{\mathcal{A}}^{2}\right]} \approx-0.0709$.

It is very instructive to look at the trading strategy exploiting the arbitrage discussed in Proposition 4. 1. In discrete time, the initial value of this portfolio is $V(0)=\sum_{\mu} \phi_{\mu}(0) X_{\mu}(0)=$ 0 and the value at time $t$ is simply

$$
V(t)=\sum_{s=0}^{t-1} \sum_{A, \mu} \hat{\alpha}^{A}(s) \hat{J}_{\mu}^{A}(s) \frac{X_{\mu}(s+1)-X_{\mu}(s)}{X_{\mu}(s)}=\sum_{s=0}^{t-1} \hat{\mathcal{A}}^{2}(s+1) .
$$

In figure 9 we show the value of this portfolio for the daily data of the three US indexes. We include the integrated profit and losses of the indexes themselves for comparison. We have multiplied the index signals by a numerical factor so that it fits in the same picture. Therefore, the overall scale in the y-axis is irrelevant. We can see that, as expected, that the performance of this portfolio is very poor for such low frequency data.

Next we look at the same index set (DJA, IXIC, NYA), but now at short time scales. As an example, we study high frequency data obtained on July 28, 2009. The data points have 7 to 10 seconds in separation. The data was collected using the "FinancialData" package of Mathematica. The gauge invariant matrix $\hat{G}$ has been estimated using a moving window of 500 data points. We have also assumed one null direction $(k=1)$. A sample of the arbitrage detection algorithm is shown in figure 10. It is quite obvious from this figure that the signal has a very significant positive skewness. In fact, a prominent feature of the signal is a series of positive peaks. These transient events have a duration of the order of 5-10 time steps, 


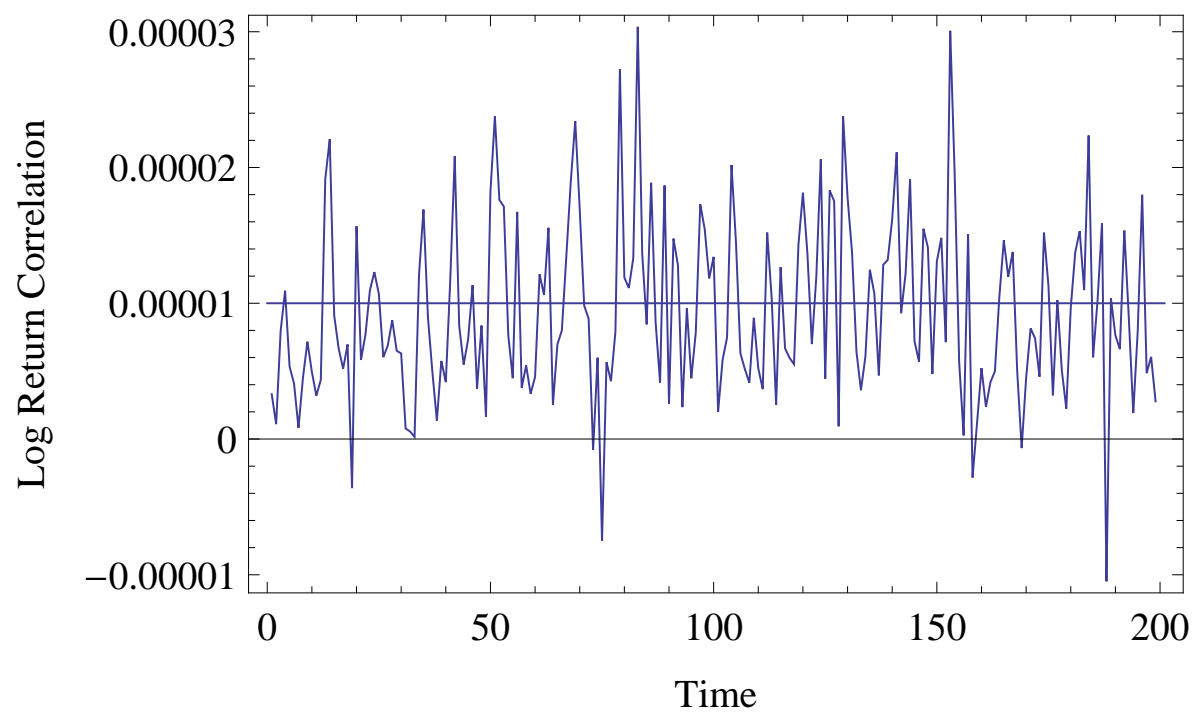

Figure 6: Product of subsequent log returns in the presence of microstructure noise, according to Eq. (115). The average of this signal is equal to $\eta^{2}=10^{-5}$ (horizontal line) in agreement with the theoretical prediction.

which for this data is about one minute. The amplitude of the peaks is quite significant compared to the noise. We argue that these peaks are precisely temporary fluctuations with $\mathcal{A}^{2} \neq 0$, that is, non-zero curvature events in the market. To show that these are not isolated events, figure 11 shows the histogram for the full data sample. We can see significant positive skewness in the signal, compared to the daily data (c.f. figure 7 ). In fact, we find a significant signal to noise ratio: $\mathbb{E}\left[\hat{\mathcal{A}}^{2}\right] / \sqrt{\operatorname{Var}\left[\hat{\mathcal{A}}^{2}\right]} \approx 0.32$. The integrated profit and losses of the arbitrage porfolio of Eq. (117) is shown in figure 12. We can see a very good performance in comparison with the daily data (c.f. figure 9). Because of model risk, such portfolio can indeed have a finite probability of a loss on short time scales. However, we see that on longer scales (integrated signal), the probability of a loss goes to zero asymptotically as $t \rightarrow \infty$. This is an example of a statistical arbitrage as discussed in [Po07, Bo03.

We have also studied the effect of the microstructure noise on the high frequency signal. In particular, we have computed the estimate of the noise $\hat{\eta}^{2}$ defined in Eq. (115). We have found that for this particular data sample, the contribution from such noise is very low: $\mathbb{E}\left[\hat{\eta}^{2}\right] / \sqrt{\operatorname{Var}\left[\hat{\eta}^{2}\right]} \approx 0.04$. The effect becomes quite significant, however, if we look at traded assets such as stocks and futures.

Our next data sample consist of the following set of US index futures: E-Mini S\&P 500 (ESU09.CME), DJIA mini-sized (YMU09.CBT), E-Mini Nasdaq 100 (NQU09.CME) and S\&P 500 Index Future (SPU09.CME). The data was collected on August 9, 2009, and all futures expire on September 2009. We have collected prices with a frequency of 7-10 seconds 


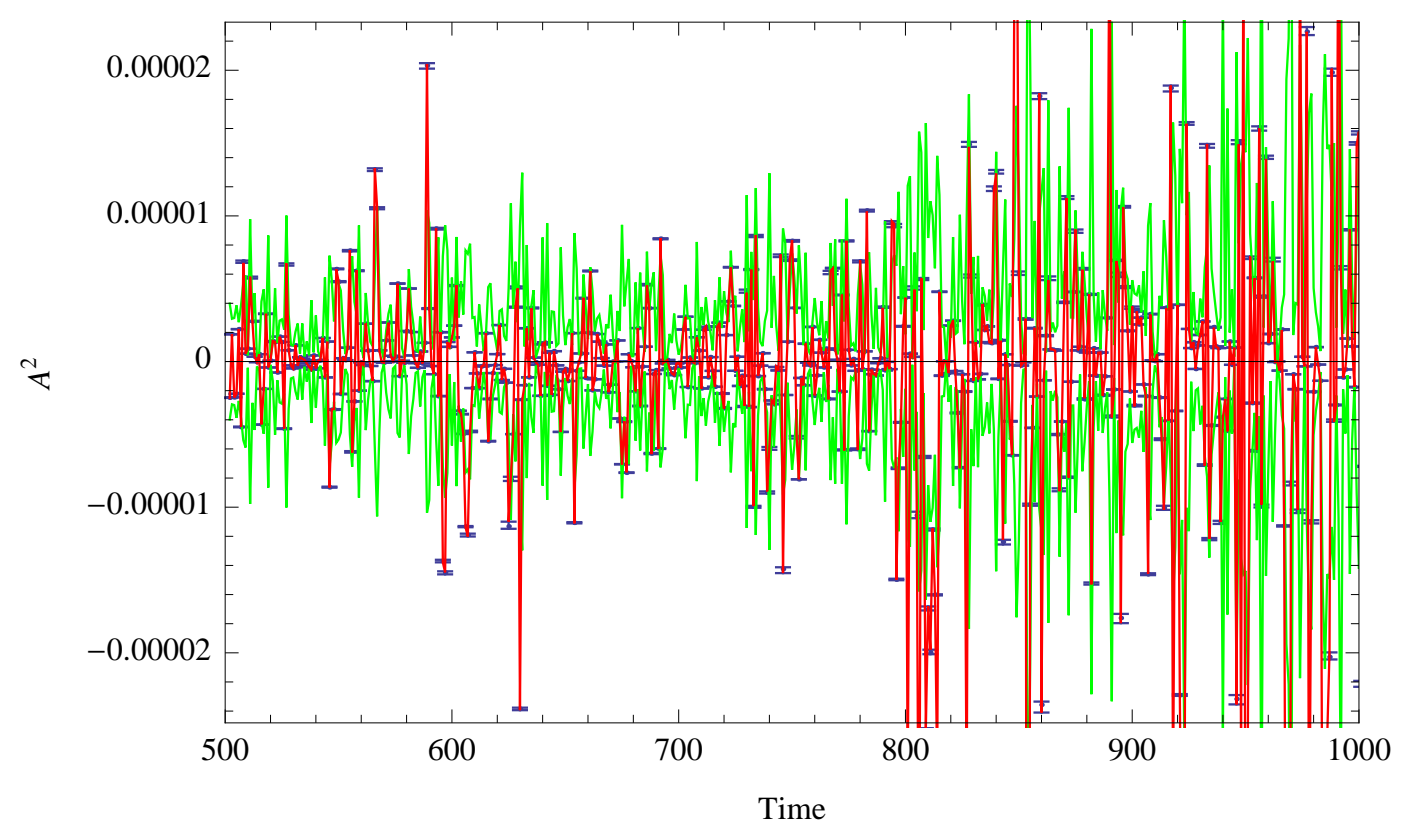

Figure 7: Arbitrage detection algorithm applied to daily closing prices of 3 major US indexes: DJA, IXIC and NYA. Here we show a sample of 500 data points. The green lines are an estimate of the variance of the gauge invariant noise using Eq. (116). The error bars give the range of values obtained using the different gauges. The solid dots are the mean of all results. The red line is the USD signal.

separation, using the "FinancialData" package of Mathematica. These securities are highly correlated. Therefore, they are ideal for the search of the arbitrage signal. However, since these are traded instruments, the effect of the bid/ask spread is more pronounced. In figure 13 we show the histogram of the values of $\hat{\mathcal{A}}^{2}$ obtained by applying exactly the same algorithm as in the previous example. We get a very poor signal; in fact, we get a negative mean: $\mathbb{E}\left[\hat{\mathcal{A}}^{2}\right] / \sqrt{\operatorname{Var}\left[\hat{\mathcal{A}}^{2}\right]} \approx-0.061$. The integrated profit and losses of the simple portfolio of Eq. (117) are shown in figure 14.

We have also calculated the effect of the microstructure noise, by computing the estimate $\hat{\eta}^{2}$ defined in Eq. (115). The effect for this data sample is about an order of magnitude bigger than the previous example: $\mathbb{E}\left[\hat{\eta}^{2}\right] / \sqrt{\operatorname{Var}\left[\hat{\eta}^{2}\right]} \approx 0.15$. This noise is the main obstacle to a detection of $\mathcal{A}^{2}$. Nevertheless, one can devise more complicated detection methods which filter out the microstructure noise. In figure 14 we show the integrated profit and losses of a particular proprietary strategy. A detailed study of such strategies is beyond the scope of this paper. 


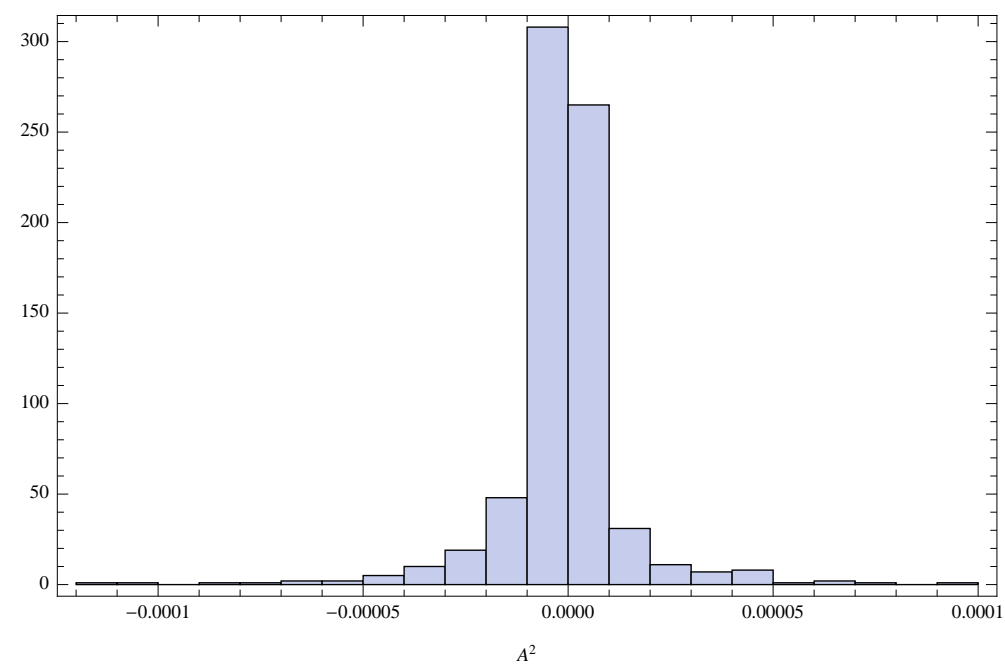

Figure 8: Histogram of different values of $\hat{\mathcal{A}}^{2}$ obtained using daily market data for DJA, IXIC and NYA. The signal to noise ratio is: $\mathbb{E}\left[\hat{\mathcal{A}}^{2}\right] / \sqrt{\operatorname{Var}\left[\hat{\mathcal{A}}^{2}\right]} \approx-0.0709$.

\section{Conclusions}

In this paper we have defined a general measure of arbitrage which is invariant under changes of numéraire and equivalent probability measure. Our main assumption is that all financial instruments can be described by Itô processes. This is not a very strong assumption as many complex financial models, including those reflecting the non-Gaussian nature of stock returns, can be modeled this way. We showed that the gauge invariant arbitrage measure can be interpreted in terms of the curvature of the stochastic version of the Malaney-Weinstein connection ([Ma96], We06]). The zero curvature condition is then equivalent to the noarbitrage principle. Moreover, we demonstrated a simple generalization of the classic asset pricing theorem to include arbitrage. Finally, we have presented a basic algorithm to measure the market curvature using financial data. We found evidence for non-zero curvature fluctuations in high frequency data involving stock indexes and index futures.

From a financial perspective, our algorithms can be used to exploit arbitrage systematically, and generate profitable trading strategies. This will require much more empirical research, and the development of more sophisticated techniques to estimate the arbitrage curvature measure $\mathcal{A}^{2}$. This is left for future work.

From a scientific perspective, we believe that our findings represent a modest step towards the understanding of the non-equilibrium market dynamics. Gauge theories provide the natural mathematical language to that aim, and arbitrage opportunities can be interpreted as a non-zero curvature fluctuation in an economy out of equilibrium. It is interesting how most of our current economic and financial thinking rely so much on the assumptions of 


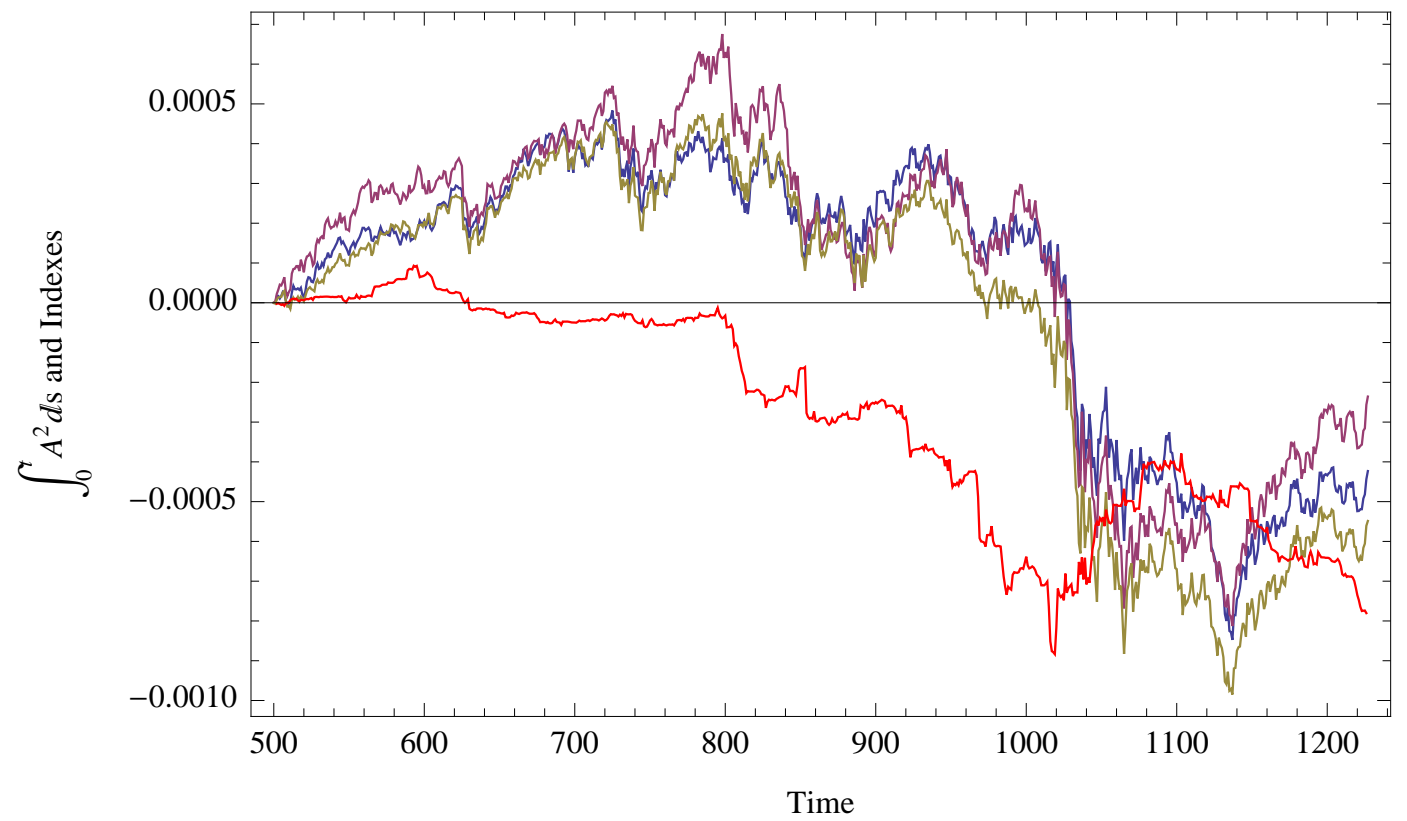

Figure 9: Integrated profit and losses of the arbitrage portfolio (red line) for the daily data of the US stock indexes DJA, IXIC and NYA. We also show the integrated profit and losses of the indexes themselves.

general equilibrium theory.

There has been a growing consensus that we need a better understanding of the nonequilibrium dynamics of the economy (see e.g. [FaGe08]). In particular, one would like to understand what is the relaxation time scale for non-equilibrium fluctuations to disappear (if they do). Within the limited data sample that we have shown in this paper, the relaxation time seems to be of the order of one minute. However, this can be very different in other sectors of the market.

\section{Acknowledgements}

We would like to extend our gratitude to Eric Weinstein, Pia Malaney, Lee Smolin, Bernd Schroers, Mike Brown, Simone Severini, Jiri Hoogland, Jim Herriot, and Bruce Sawhill for many discussions and ideas which influenced the results of this paper. Research at Perimeter Institute is supported by the Government of Canada through Industry Canada and by the Province of Ontario through the Ministry of Research \& Innovation. 


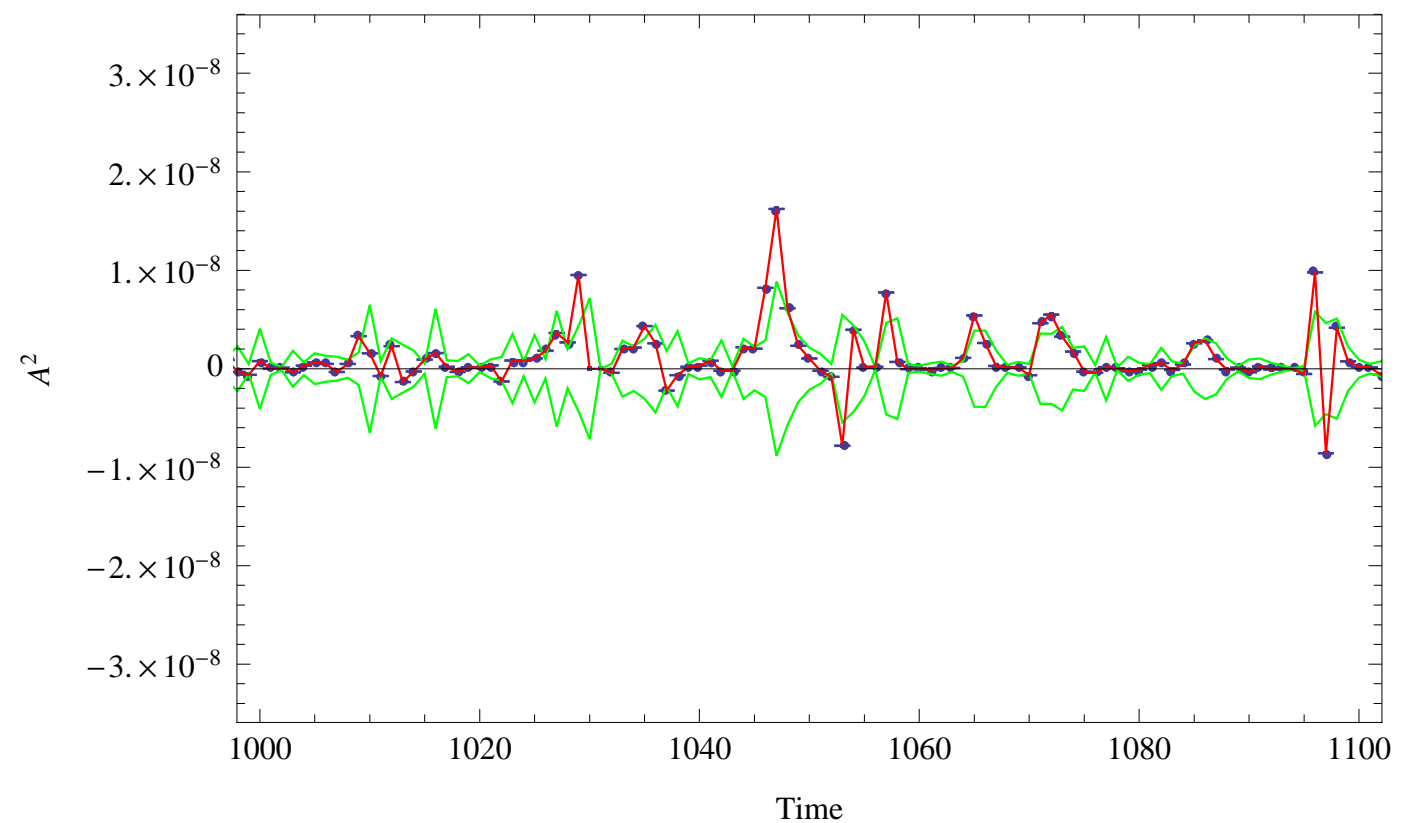

Figure 10: Arbitrage detection algorithm applied to high frequency data for 3 major US indexes: DJA, IXIC and NYA. Here we show a sample of 100 data points. The green lines are an estimate of the variance of the gauge invariant noise using Eq. (116). The error bars give the range of values obtained using the different gauges. The solid dots are the mean of all results. The red line is the USD signal.

\section{Appendix A}

In this appendix we consider the following general mathematical problem. Suppose we are given two $N \times N$ symmetric and real matrices which we label by $G(t)$ and $G(t+1)$ respectively. Moreover, both matrices have a null space with the same dimensionality. In other words, let $J^{A}(t)$ and $J^{A}(t+1)$ be orthonormal basis vectors such that

$$
G(t) J^{A}(t)=0, \quad A=1, \ldots, k, \quad G(t+1) J^{B}(t+1)=0, \quad B=1, \ldots, k^{\prime},
$$

and $k=k^{\prime}$. Now let $\|\cdot\|$ be some matrix norm such that $\|G(t)-G(t+1)\| \ll 1$. More precisely, we are interested in the limit in which both matrices are very similar to each other. We then wish to find a $k \times k$ rotation matrix $C$ such that

$$
\lim _{\|G(t)-G(t+1)\| \rightarrow 0} J^{A}(t+1)=\sum_{B} C^{A B} J^{B}(t), \quad C^{\dagger} C=\mathbf{1} .
$$

In physics, this problem arises in the so-called degenerate perturbation theory [Sa94]. However, it is often assumed that $G(t+1)$ will "lift the degeneracy", which in our case means 


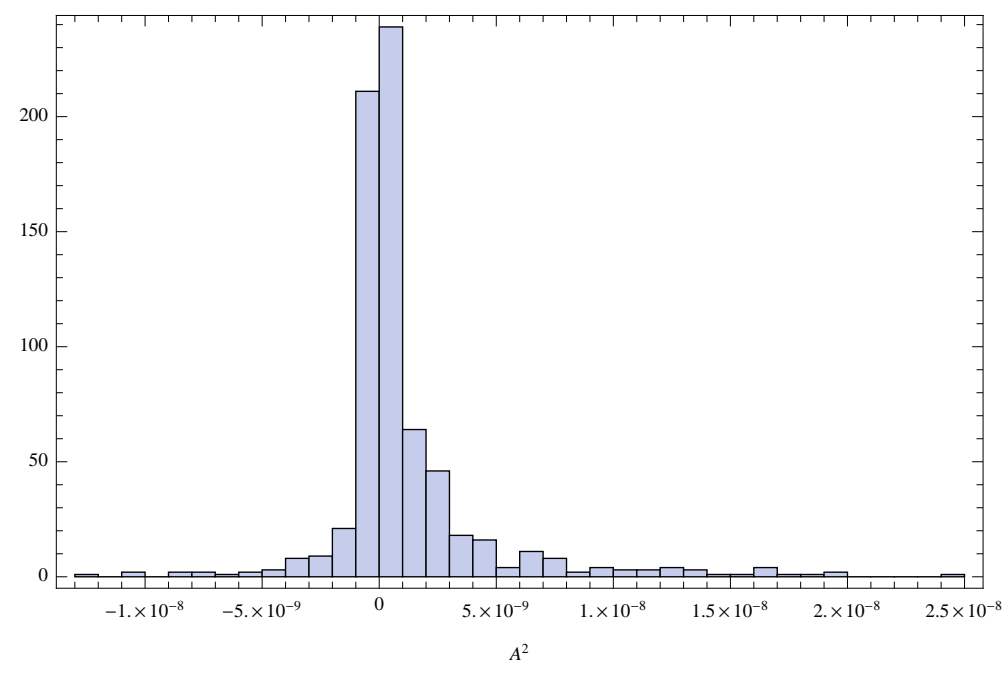

Figure 11: Histogram of $\hat{\mathcal{A}}^{2}$ obtained using high frequency data for DJA, IXIC and NYA. The signal to noise ratio is: $\mathbb{E}\left[\hat{\mathcal{A}}^{2}\right] / \sqrt{\operatorname{Var}\left[\hat{\mathcal{A}}^{2}\right]} \approx 0.32$.

$k^{\prime}<k$. This is not the case in our problem, and so the usual perturbation theory methods do not work.

We are interested in solving the problem above only approximately. In fact, in practice we have no control over $G(t)$ and $G(t+1)$, and so we cannot take the limit $\|G(t)-G(t+1)\| \rightarrow 0$ explicitly. However, we will assume that $\|G(t)-G(t+1)\| \ll 1$ (which is true most of the time). Now consider the $k \times k$ real matrix $\tilde{C}$ with components

$$
\tilde{C}^{A B}:=\left[J^{A}(t+1)\right]^{\dagger} J^{B}(t)
$$

Under our assumptions, we have

$$
\tilde{C}^{A B} \approx C^{A B}+\mathcal{O}(\delta G)
$$

where $\delta G=G(t)-G(t+1)$. Therefore, we seek a rotation matrix $\hat{C}$ which approximates $\tilde{C}$ as closely as possible. In the limit $\|G(t)-G(t+1)\| \rightarrow 0$ we should have $\hat{C} \rightarrow C$. In other words, $\hat{C}$ will be the our best approximation to $C$.

We can solve this problem using Lagrange multipliers. We seek to minimize the Lagrangian

$$
\mathcal{L}=\operatorname{Tr}\left[(\tilde{C}-\hat{C})^{\dagger}(\tilde{C}-\hat{C})\right]+\operatorname{Tr}\left[\lambda\left(\hat{C}^{T} \hat{C}-\mathbf{1}\right)\right],
$$

where $\lambda$ is a symmetric matrix which serves as a Lagrange multiplier implementing the 


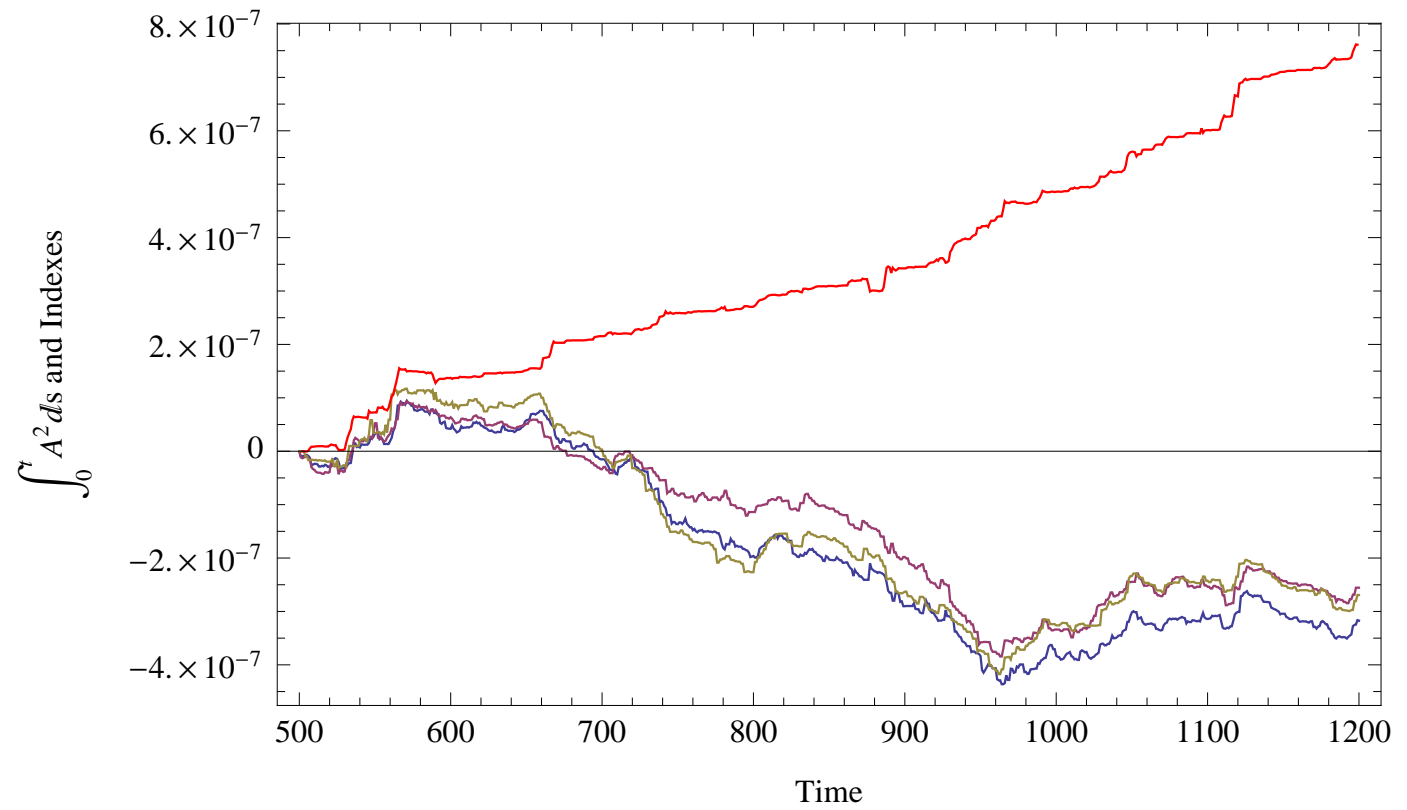

Figure 12: Integrated profit and losses of the arbitrage portfolio (red line) for the high frequency data of the US stock indexes DJA, IXIC and NYA. We also show the integrated profit and losses of the indexes themselves.

constraint $\hat{C}^{T} \hat{C}=1$. The Lagrange equations are then

$$
\frac{\partial \mathcal{L}}{\partial \hat{C}^{A B}}=0, \quad \frac{\partial \mathcal{L}}{\partial \lambda^{A B}}=0 .
$$

We will only present the solution to Eqs. (123) for the cases of $k=1$ and $k=2$.

For $k=1$, the rotation matrix $\hat{C}$ is simply a sign and one can show that the minimum of $\mathcal{L}$ is

$$
\hat{C}=\operatorname{sign}(\tilde{C}) .
$$

For $k=2$, the solution can be written in terms of the Pauli matrices:

$$
\sigma_{0}:=\left(\begin{array}{ll}
1 & 0 \\
0 & 1
\end{array}\right), \quad \sigma_{1}:=\left(\begin{array}{ll}
0 & 1 \\
1 & 0
\end{array}\right), \quad \sigma_{2}:=\left(\begin{array}{cc}
1 & 0 \\
0 & -1
\end{array}\right), \quad \sigma_{3}:=\left(\begin{array}{cc}
0 & 1 \\
-1 & 0
\end{array}\right) .
$$

We can expand,

$$
\tilde{C}=\sum_{i=0}^{3} \tilde{c}_{i} \sigma_{i}, \quad \hat{C}=\sum_{i=0}^{3} \hat{c}_{i} \sigma_{i}, \quad \lambda=\sum_{i=0}^{2} \lambda_{i} \sigma_{i},
$$

where

$$
\tilde{c}_{i}=\frac{1}{2} \operatorname{Tr}\left(\sigma_{i}^{\dagger} \tilde{C}\right), \quad i=0, \ldots, 3,
$$




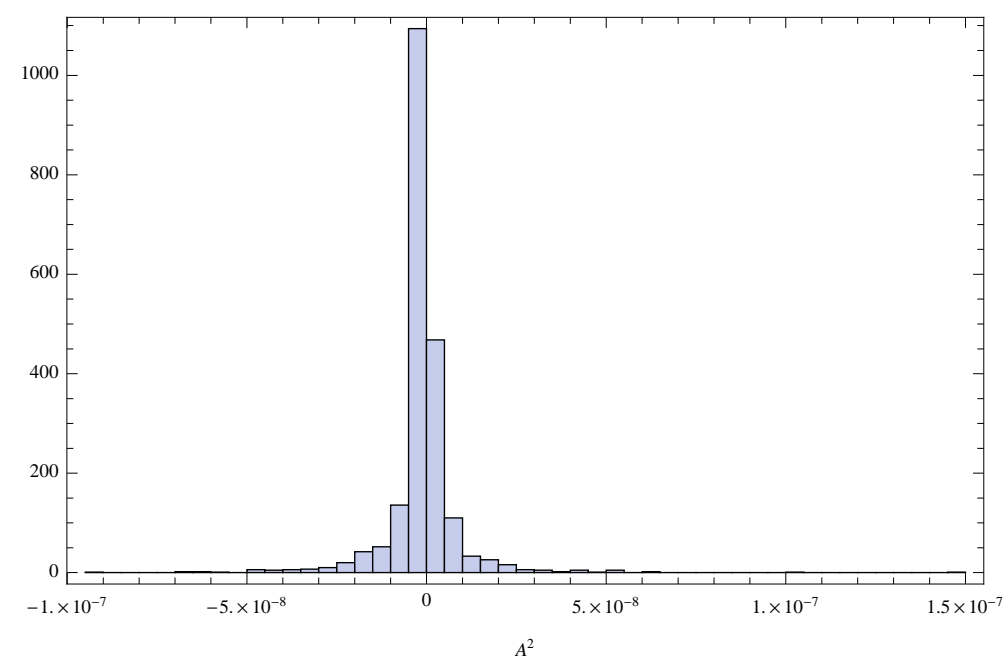

Figure 13: Histogram of $\hat{\mathcal{A}}^{2}$ obtained using high frequency data for the index futures: ESU09.CME, YMU09.CBT, NQU09.CME, SPU09.CME. The signal to noise ratio is: $\mathbb{E}\left[\hat{\mathcal{A}}^{2}\right] / \sqrt{\operatorname{Var}\left[\hat{\mathcal{A}}^{2}\right]} \approx$ -0.061. This picture illustrates the negative effects of the market microstructure noise for the simplest trading strategy.

and similarly for $\hat{c}_{i}$ and $\lambda_{i}$. The solution to the Lagrange equations, Eq. (123), which correspond to the minimum of $\mathcal{L}$ can be shown to be

$$
\hat{c}_{0}=\hat{c}_{3}=0, \quad \hat{c}_{1}=\frac{\tilde{c}_{1}}{\sqrt{\tilde{c}_{1}^{2}+\tilde{c}_{2}^{2}}}, \quad \hat{c}_{2}=\frac{\tilde{c}_{2}}{\sqrt{\tilde{c}_{1}^{2}+\tilde{c}_{2}^{2}}},
$$

for $\tilde{c}_{1}^{2}+\tilde{c}_{2}^{2}>\tilde{c}_{0}^{2}+\tilde{c}_{3}^{2}$, and

$$
\hat{c}_{1}=\hat{c}_{2}=0, \quad \hat{c}_{0}=\frac{\tilde{c}_{0}}{\sqrt{\tilde{c}_{0}^{2}+\tilde{c}_{3}^{2}}}, \quad \hat{c}_{3}=\frac{\tilde{c}_{3}}{\sqrt{\tilde{c}_{0}^{2}+\tilde{c}_{3}^{2}}},
$$

otherwise.

\section{References}

[AcTi99] L. F. ACKERT, and Y. S. TIAN, Efficiency in Index Options Markets and Trading in Stock Baskets, Federal Reserve Bank of Atlanta Working Paper 99-5, 1999.

[Bo03] O. BONDARENKO, Statistical Arbitrage and Securities Prices, The Review of Financial Studies, 16, 875, (2003).

[CvZa04] J. CVITANIC and F. ZAPATERO Introduction to the Economics and Mathematics of Financial Markets, The MIT Press, 2004. 


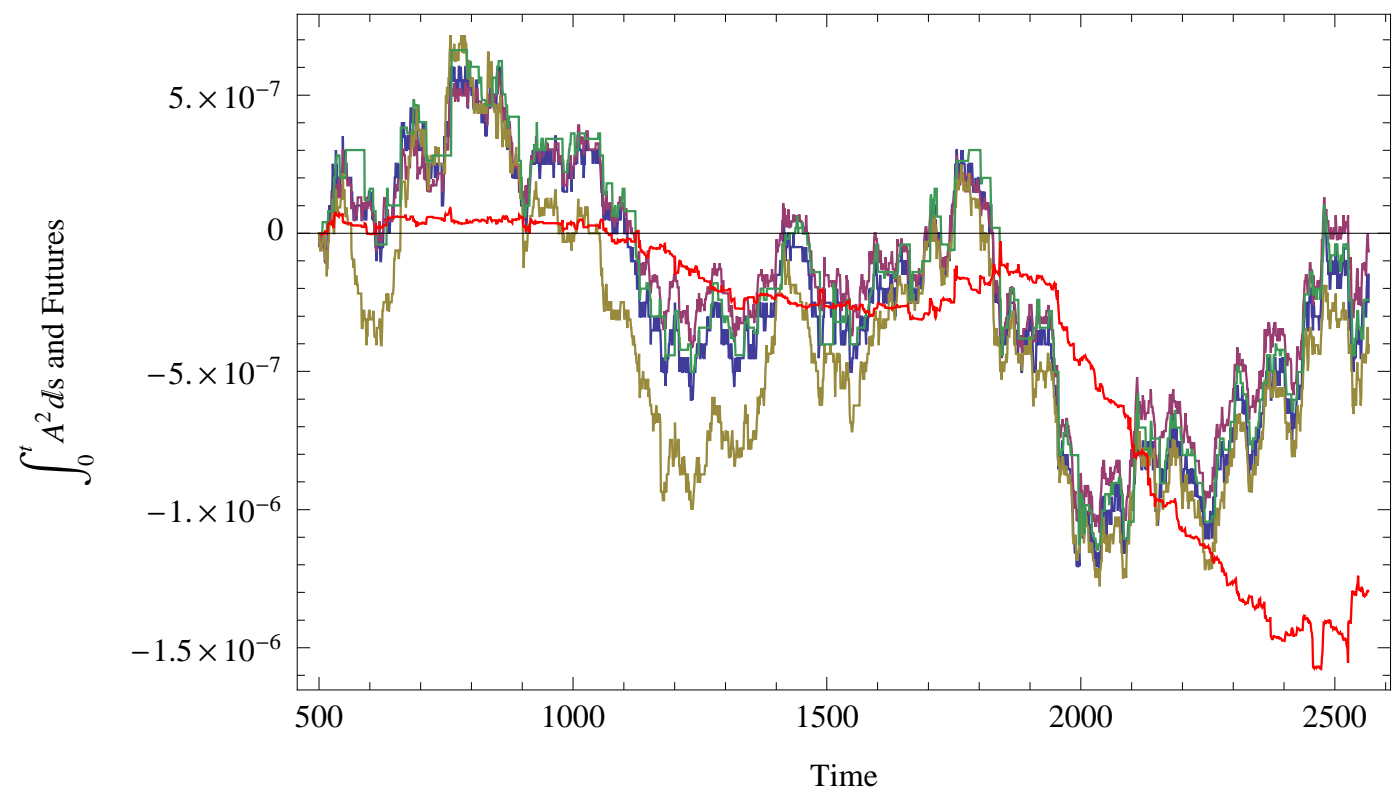

Figure 14: Integrated profit and losses of the arbitrage portfolio (red line) of Eq. 117) for the high frequency data of the US index futures: ESU09.CME, YMU09.CBT, NQU09.CME, SPU09.CME. We also show the integrated profit and losses of the futures themselves. This picture illustrates the negative effects of the market microstructure noise for the simplest trading strategy.

[DeSc08] F. DELBAEN and W. SCHACHERMAYER The Mathematics of Arbitrage, Springer 2008.

[Fam98] E. F. FAMA, Market Efficiency, Long-Term Returns, and Behavioral Finance, Journal of Financial Economics, 49, 283, 1998.

[Far08] S. FARINELLI, Geometric Arbitrage Theory and Calibration of a Generator of Consistent Economic Scenarios, Electronic copy available at: http://ssrn.com/abstract=1115860, 2008.

[Far09a] S. FARINELLI, Geometric Arbitrage Theory and Market Dynamics, Electronic copy available at: http://ssrn.com/abstract=1113292, 2009.

[Far09b] S. FARINELLI, Stress Test and Consistent Aggregation of Market, Credit and Transfer Risk by Geometric Arbitrage Theory, Electronic copy available at: http://ssrn.com/abstract=1103882, 2009.

[FaGe08] J. D. FARMER and J. GEANAKOPLOS, The Virtues and Vices of Equilibrium and the Future of Financial Economics, Electronic version available online at: arxiv.org/abs/0803.2996, 2008. 


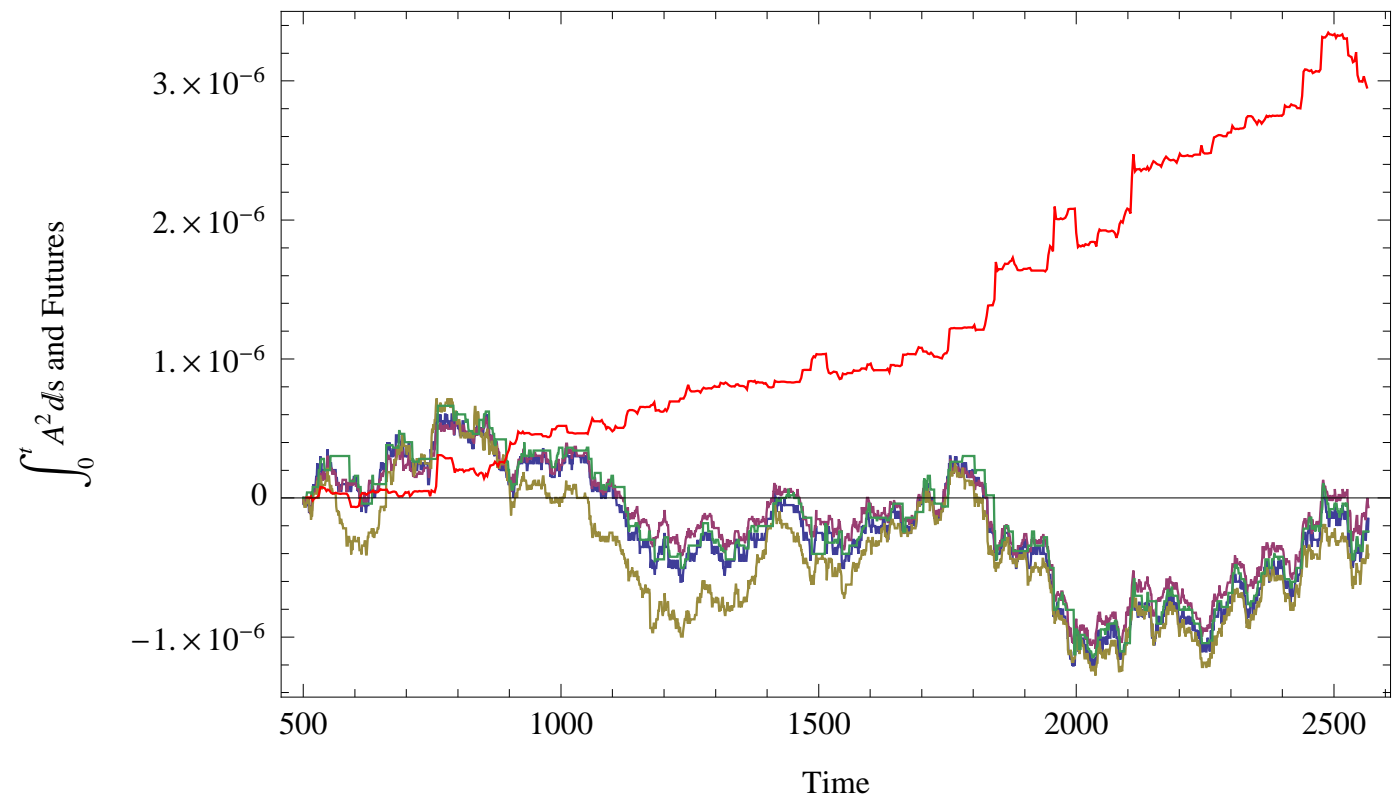

Figure 15: Integrated profit and losses of a proprietary arbitrage portfolio (red line) for the high frequency data of the US index futures: ESU09.CME, YMU09.CBT, NQU09.CME, SPU09.CME. We also show the integrated profit and losses of the futures themselves. This particular strategy is designed to minimize the effects of the microstructure noise.

[GaGoRo06] E. GATEV, W. N. GOETZMAnN, and K. G. ROUWENHORST, Pairs Trading: Performance of a Relative-Value Arbitrage Rule, The Review of Financial Studies, 19, 797, 2006.

[HaHaPi08] W. HARDLE, N. HAUTSCH and U. PIGORSCH, Measuring and Modeling Risk Using High-Frequency Data, SFB 649 Discussion Paper 2008-045. Available online at: sfb649.wiwi.hu-berlin.de, 2008.

[HoNe99a] J. HOOGLAND and D. NEUMANN, Scale-invariance and contingent claim pricing, arXiv.org:cond-mat/9906048, 1999.

[HoNe99b] J. HOOGLAND and D. NEUMANN, Scaling invariance in finance II: Pathdependent contingent claims, arXiv.org:cond-mat/9907185, 1999.

[HoNe00] J. HOOGLAND and D. NEUMANN, Asians and cash dividends: Exploiting symmetries in pricing theory, arXiv.org:cond-mat/0006133, 2000.

[HoNeVe01] J. HOOGLAND, D. NEUMANN and M. VELLEKOOP, Symmetries in jump-diffusion models with applications in option pricing and credit risk, arXiv.org:cond-mat/0108137, 2001. 
[I197] K. ILLINSKI, Black-Scholes equation from Gauge Theory of Arbitrage", pre-print available at arxiv.org/abs/hep-th/9712034, 1997.

[Il01] K. ILINSKI, Physics of Finance: Gauge Modelling in Non-Equilibrium Pricing, Wiley, 2001.

[JeTi93] N. JEGADEESH and S. TITMAN, Returns to Buying Winners and Selling Losers: Implications for Stock Market Efficiency, Journal of Finance, 48, 65, 1993.

[La08] P.-H. LABORDÈRE, Analysis, Geometry, and Modeling in Finance: Advanced Methods in Option Pricing, Chapman \& Hall/CRC Financial Mathematics Series, 2008.

[LaLa06] D. LAMBERTON and B. LAPEYRE Introduction to Stochastic Calculus Applied to Finance, Second Edition, Chapman \& Hall/Crc Financial Mathematics Series, 2007.

[Ma96] P. N. MALANEY, The Index Number Problem: A Differential Geometric Approach, PhD Thesis, Harvard University Economics Department, 1996.

[Ma03] B. G. MALKIEL, The Efficient Market Hypothesis and its Critics, Journal of Economic Perspectives, 17, 59, 2003.

[MuRu07] M. MUSIELA AND M. RUTKOWSKI, Martingale Methods in Financial Modeling, Second Edition, Stochastic Modeling And Applied Probability 36, Springer, 2007.

[Po07] A. POLE, Statistical Arbitrage: Algorithmic Trading Insights and Techniques, Wiley Finance, 2007.

[Sa94] J. J. SAKURAI, Modern Quantum Mechanics, Revised Ed., Addison-Wesley Publishing, 1994.

[ShVi97] A. SHLEIFER, and R. W. VISHNY, The Limits of Arbitrage, The Journal of Finance, 52, 35, (1997).

[Sh00] S. E. SHREVE, Stochastic Calculus for Finance”, Vols. I and II, Springer, 2000.

[Sm09] L. SMOLIN, Time and symmetry in models of economic markets, Electronic version available online at: arxiv.org/abs/0902.4274, 2009.

[So06] D. SONDERMANN, Introduction to Stochastic Calculus for Finance: A New Didactic Approach, Lecture Notes in Economics and Mathematical Systems 579, Springer, 2006. 
[VKr81] N. G. VAN KRAMPEN, Itô Versus Stratonovich, J. Stat. Phys., 24, 175, 1981.

[We06] E. WEINSTEIN, Gauge Theory and Inflation: Enlarging the Wu-Yang Dictionary to a unifying Rosetta Stone for Geometry in Application, Talk given at Perimeter Institute, 2006. Available online at: http://pirsa.org/06050010/, 2006.

[Y099] K. YOUNG, Foreign Exchange Market as a Lattice Gauge Theory, Am. J. Phys. 67, 1999.

[Zh06] L. ZHANG, Efficient Estimation of Stochastic Volatility Using Noisy Observations: A Multi-Scale Approach, Electronic version available online at: arxiv.org/abs/math/0411397, 2006. 